\title{
An Approach to the Synthesis of Thioesters and Selenoesters Promoted by Rongalite $^{\circledR}$
}

\author{
Shao-Miao Lin, ${ }^{a}$ Ji-Lei Zhang, ${ }^{a}$ Jiu-Xi Chen, $*, a$ Wen-Xia Gao, ${ }^{a}$ Jin-Chang Ding, ${ }^{a, b}$ \\ Wei-Ke Su ${ }^{a, c}$ and Hua-Yue $\mathrm{Wu}^{*, a}$
}

\author{
${ }^{a}$ College of Chemistry and Materials Engineering, Wenzhou University, 325035 Wenzhou, P. R. China \\ ${ }^{b}$ Wenzhou Vocational and Technical College, 325035 Wenzhou, P. R. China
}

\author{
'Zhejiang Key Laboratory of Pharmaceutical Engineering, College of Pharmaceutical Sciences, \\ Zhejiang University of Technology, 310014 Hangzhou, P. R. China
}

\begin{abstract}
Rongalite ${ }^{\circledR}$ promove a clivagem de diarildissulfetos gerando os calcogenolatos correspondentes, que reagem facilmente com $N$-acilbenzotriazóis na presença de $\mathrm{K}_{2} \mathrm{CO}_{3}$, produzindo tioésteres em bons a excelentes rendimentos. As principais características desta metodologia são a não necessidade de catalisdores metálicos ou de reagentes caros e o alto rendimento. É digno de nota que as reações de disseleneto de difenila com $N$-acilbenzotriazóis são também conduzidas para produzir selenoésteres em bons rendimentos sob condições normais.
\end{abstract}

Rongalite ${ }^{\circledR}$ promotes cleavage of diaryldisulfides generating the corresponding chalcogenolate anions that then undergo facile reaction with $\mathrm{N}$-acylbenzotriazoles in the presence of $\mathrm{K}_{2} \mathrm{CO}_{3}$ to afford thioesters in good to excellent yields. The important features of this methodology are no requirement of metal catalysts, without any expensive reagent and high yields. It is noteworthy that the reactions of diphenyl diselenide with $N$-acylbenzotriazoles are also conducted to afford selenoesters in good yields under the standard conditions.

Keywords: Rongalite ${ }^{\circledR}$, thioesters, selenoesters, $N$-acylbenzotriazoles, diaryl disulfide, diphenyldiselenide

\section{Introduction}

Thioesters have become increasingly important in the past few years because they have proven to be extremely useful intermediates for the preparation of heterocycles ${ }^{1}$ and diverse ketones, ${ }^{2}$ acyl radicals, ${ }^{3}$ and biologically active compounds. ${ }^{4}$ The typical procedure for the synthesis of thioesters involves the reaction of acylhalides with thiols, ${ }^{5}$ thiol sodium salts ${ }^{6}$ or disulfides. ${ }^{7}$ In addition, carboxylic acids are also transformed into thioesters by treatment with arylthiocyanates and tributyl phosphine in dichloromethane ${ }^{8}$ or with thiols by activated using tetramethyl fluoroformamidinium hexafluorophosphate, ${ }^{9}$ diphosgene ${ }^{10}$ and polyphosphate ester. ${ }^{11}$ Recently, Katritzky and co-workers ${ }^{12}$ introduced a new procedure for preparation of thioesters utilizing $\mathrm{N}$-acylbenzotriazoles. Some other methods include palladium-catalyzed thiocarbonylation of iodoarenes with thiols in ionic liquid, ${ }^{13}$ rhodium-catalyzed

*e-mail: jiuxichen@wzu.edu.cn, huayuewu@wzu.edu.cn alkylthio exchange reaction of thioester and disulfide, ${ }^{14}$ $\mathrm{KF} / \mathrm{Al}_{2} \mathrm{O}_{3}$-catalyzed reaction of $\mathrm{N}$-acylphthalimides with thiols ${ }^{15}$ and copper-catalyzed coupling of aryl iodides and thiobenzoic acid. ${ }^{16}$ However, these methods usually suffer from one or more limitations such as the use of unpleasant odor substrates thiols and expensive, toxic or metallic catalysts, long reaction times, unsatisfactory yields, as well as elevated temperature. Therefore, developing versatile approaches to synthesize thioesters still remains a highly desired goal in organic synthesis.

In continuation of our researches in developing novel synthetic routes for the formations of carbon-sulfur bonds ${ }^{17}$ and Rongalite ${ }^{\circledR}$-promoted organic reactions, ${ }^{18}$ we here demonstrate further extension of this work together with application of Rongalite ${ }^{\circledR}$ (sodium formaldehyde sulfoxylate, $\mathrm{HOCH}_{2} \mathrm{SO}_{2} \mathrm{Na}$ ) as an inexpensive reagent for the cleavage of diaryl disulfides or diphenyldiselenide and subsequent reaction with $\mathrm{N}$-acylbenzotriazoles $(\mathrm{Bt}=1 \mathrm{H}$ benzo[d][1,2,3]triazol-1-yl) to provide thioesters and selenoesters (Scheme 1). 


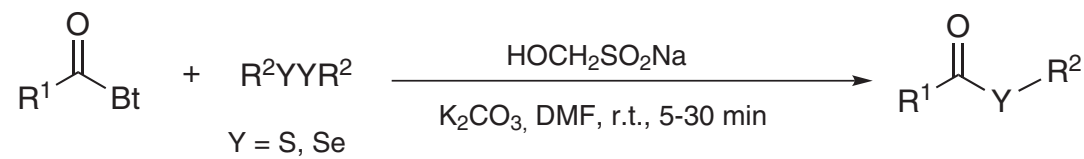

Scheme 1. Synthesis of thioesters and selenoesters.

\section{Results and Discussion}

At the onset of this work, we have investigated a variety of conditions with the model reaction of $(1 H$-benzo[d] $[1,2,3]$ triazol-1-yl)(phenyl)methanone (1a) and diphenyl disulfide (2a) using Rongalite ${ }^{\circledR}$ as promoter (Table 1). First, we examined different solvents such as toluene, $\mathrm{CH}_{2} \mathrm{Cl}_{2}, \mathrm{CH}_{3} \mathrm{CN}, \mathrm{H}_{2} \mathrm{O}, \mathrm{CH}_{3} \mathrm{CH}_{2} \mathrm{OH}$ and DMF. Among the solvents screened, it was found that DMF is a much better solvent than all others tested (Table 1, entries 1-6). Next, we evaluated the loading amount of Rongalite ${ }^{\circledR}$. No reaction was observed in the absence of Rongalite ${ }^{\circledR}$ and both starting materials were recovered in quantitative yields (Table 1, entry 7). In order to confirm, the amount of Rongalite ${ }^{\circledR}$ required for the above transformation, different experiments were carried out by varying the amount of Rongalite ${ }^{\circledR}$ (Table 1, entries 8-12). These results clearly indicate that, the use of 3 equiv. of Rongalite ${ }^{\circledR}$ is sufficient to promote the reaction in excellent yield. On the other hand, among the bases such as KF, $\mathrm{Et}_{3} \mathrm{~N}, \mathrm{~K}_{3} \mathrm{PO}_{4}$, $\mathrm{K}_{2} \mathrm{CO}_{3}$ and $\mathrm{Cs}_{2} \mathrm{CO}_{3}$ tested, $\mathrm{K}_{2} \mathrm{CO}_{3}$ was found to be the best (Table 1, entries 6 and 13-16). We also checked the effect of the amount of $\mathrm{K}_{2} \mathrm{CO}_{3}$, the desired product $3 \mathbf{a}$ was afforded in $49 \%$ without $\mathrm{K}_{2} \mathrm{CO}_{3}$ increasing the amount of $\mathrm{K}_{2} \mathrm{CO}_{3}$ to 2 equiv., it was found that 1.5 equiv. resulted in excellent yield (Table 1, entries 17-20).

Table 1. Screening conditions for the synthesis of thioesters ${ }^{\mathrm{a}}$<smiles>O=C(Br)c1ccccc1</smiles>

1a

$2 a$

3a

\begin{tabular}{lcccc}
\hline Entry & Rongalite / (equiv.) & Solvent & Base & Yield/(\%) \\
\hline 1 & 3 & toluene & $\mathrm{K}_{2} \mathrm{CO}_{3}$ & $\mathrm{NR}$ \\
2 & 3 & $\mathrm{H}_{2} \mathrm{O}$ & $\mathrm{K}_{2} \mathrm{CO}_{3}$ & $\mathrm{NR}$ \\
3 & 3 & $\mathrm{CH}_{2} \mathrm{Cl}_{2}$ & $\mathrm{~K}_{2} \mathrm{CO}_{3}$ & $\mathrm{NR}$ \\
4 & 3 & $\mathrm{CH}_{3} \mathrm{CH}_{2} \mathrm{OH}$ & $\mathrm{K}_{2} \mathrm{CO}_{3}$ & trace \\
5 & 3 & $\mathrm{CH}_{3} \mathrm{CN}$ & $\mathrm{K}_{2} \mathrm{CO}_{3}$ & 10 \\
6 & 3 & $\mathrm{KMF}$ & 92 \\
7 & $-\mathrm{K}_{3}$ & - \\
8 & - & $\mathrm{DMF}$ & $\mathrm{K}_{2} \mathrm{CO}_{3}$ & 70 \\
10 & 1 & $\mathrm{KMF}$ & $\mathrm{K}_{2} \mathrm{CO}_{3}$ & 86 \\
11 & 2 & $\mathrm{~K}_{2} \mathrm{CO}_{3}$ & 93 \\
12 & $\mathrm{DMF}$ & $\mathrm{K}_{2} \mathrm{CO}_{3}$ & 89 \\
13 & 4 & $\mathrm{~K}_{2} \mathrm{CO}_{3}$ & 67 \\
14 & 5 & $\mathrm{KF}_{2} \mathrm{H}_{2} \mathrm{O}$ & 85 \\
15 & 3 & $\mathrm{DMF}$ & $\mathrm{Et}_{3}$ & 84 \\
16 & 3 & $\mathrm{~K}_{3} \mathrm{PO}_{4}$ & 88 \\
17 & 3 & $\mathrm{DMF}$ & $\mathrm{Cs}_{2} \mathrm{CO}_{3}$ & $49^{\mathrm{c}}$ \\
18 & 3 & $\mathrm{DMF}$ & $\mathrm{K}_{2} \mathrm{CO}_{3}$ & $81^{\mathrm{d}}$ \\
19 & 3 & $\mathrm{~K}_{2} \mathrm{CO}_{3}$ & $87^{\mathrm{e}}$ \\
20 & 3 & $\mathrm{DMF}$ & $\mathrm{K}_{2} \mathrm{CO}_{3}$ & $86^{\mathrm{f}}$ \\
\hline
\end{tabular}

Reaction conditions: $1 \mathrm{H}$-1,2,3-benzotriazol-1-yl (phenyl) methanone 1a (0.4 mmol), 1,2-diphenyldisulfane $\mathbf{2 a}(0.2 \mathrm{mmol}), \mathrm{HOCH}_{2} \mathrm{SO}_{2} \mathrm{Na}_{2}$ base, solvent, r.t., 5 min. ${ }^{b}$ Isolated yields. ${ }^{~}$ Without $\mathrm{K}_{2} \mathrm{CO}_{3} \cdot{ }^{\mathrm{d}} 0.5$ equiv. of $\mathrm{K}_{2} \mathrm{CO}_{3} \cdot{ }^{\mathrm{e}} 1$ equiv. of $\mathrm{K}_{2} \mathrm{CO}_{3} \cdot{ }^{\text {f }} 2$ equiv. of $\mathrm{K}_{2} \mathrm{CO}_{3} \cdot \mathrm{NR}=$ No Reaction. 
With the optimal conditions in hand, the scope of both disulfides and $N$-acylbenzotriazoles were explored and the results are summarized in (Table 2). As expected, this reaction proceeded smoothly and the desired products were afforded in good to excellent yields. A series of $\mathrm{N}$-acylbenzotriazoles with either electron-donating or electron-withdrawing groups attached to aromatic ring were investigated. The substitution groups on the aromatic ring had no obvious effect on the yield. We also examined reaction of aliphatic $N$-acylbenzotriazole such as 1-(1H-benzo[d][1,2,3]triazol-1-yl)propan-1-one (1d), the desired products of $\mathbf{3 j}$-3l were afforded in good yields (Table 2, entries 10-12). Similarly, $\mathbf{3 m}$ and $\mathbf{3 n}$ were afforded from heterocyclic $N$-acylbenzotriazole such as $(1 H$-benzo[d] [1,2,3]triazol-1-yl) (furan-2-yl)methanone (1e) in moderate yields (Table 2, entries 13-14). Unfortunately, attempt to acylation of dibenzyl disulfide, an aliphatic disulfide, with (1H-benzo[d][1,2,3]triazol-1-yl)(phenyl)methanone (1a) failed to give the expected thioesters.

Interestingly, when cinnamic $N$-acylbenzotriazole (1f) was used, the corresponding multi-sulfur substitution compounds of $\mathbf{4 a - 4} \mathbf{c}$ were afforded in moderate yields (Scheme 2).

On the other hand, we extended this method to prepare selenoesters starting from $\mathrm{N}$-acylbenzotriazoles. Selenoesters are important intermediates in several organic

Table 2. Synthesis of diverse thiol esters from $N$-acylbenzotriazoles with disulfides ${ }^{\text {a }}$

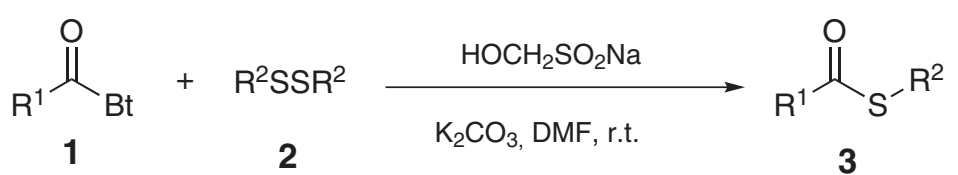

\begin{tabular}{|c|c|c|c|c|c|}
\hline Entry & $\mathrm{R}^{1}(\mathbf{1})$ & $\mathrm{R}^{2}$ & time / min & Product & Yield / $(\%)^{t}$ \\
\hline 1 & $\mathrm{C}_{6} \mathrm{H}_{5}(\mathbf{1} \mathbf{a})$ & $\mathrm{C}_{6} \mathrm{H}_{5}$ & 5 & 3a & 92 \\
\hline 2 & $\mathrm{C}_{6} \mathrm{H}_{5}(\mathbf{1} \mathbf{a})$ & $p$-(Me) $\mathrm{C}_{6} \mathrm{H}_{4}$ & 5 & $3 \mathbf{b}$ & 95 \\
\hline 3 & $\mathrm{C}_{6} \mathrm{H}_{5}(\mathbf{1} \mathbf{a})$ & $p-(\mathrm{Cl}) \mathrm{C}_{6} \mathrm{H}_{4}$ & 5 & $3 c$ & 90 \\
\hline 4 & $p-(\mathrm{MeO}) \mathrm{C}_{6} \mathrm{H}_{4}(\mathbf{1 b})$ & $\mathrm{C}_{6} \mathrm{H}_{5}$ & 5 & $3 d$ & 97 \\
\hline 5 & $p-(\mathrm{MeO}) \mathrm{C}_{6} \mathrm{H}_{4}(\mathbf{1 b})$ & $p$-(Me) $\mathrm{C}_{6} \mathrm{H}_{4}$ & 5 & $3 e$ & 93 \\
\hline 6 & $p-(\mathrm{MeO}) \mathrm{C}_{6} \mathrm{H}_{4}(\mathbf{1 b})$ & $p-(\mathrm{Cl}) \mathrm{C}_{6} \mathrm{H}_{4}$ & 5 & $3 f$ & 98 \\
\hline 7 & $o-(\mathrm{I}) \mathrm{C}_{6} \mathrm{H}_{4}(\mathbf{1} \mathbf{c})$ & $\mathrm{C}_{6} \mathrm{H}_{5}$ & 15 & $3 g$ & 92 \\
\hline 8 & $o$-(I) $\mathrm{C}_{6} \mathrm{H}_{4}(\mathbf{1} \mathbf{c})$ & $p$-(Me) $\mathrm{C}_{6} \mathrm{H}_{4}$ & 15 & $3 \mathrm{~h}$ & 93 \\
\hline 9 & $o-(\mathrm{I}) \mathrm{C}_{6} \mathrm{H}_{4}(\mathbf{1} \mathbf{c})$ & $p-(\mathrm{Cl}) \mathrm{C}_{6} \mathrm{H}_{4}$ & 15 & $3 \mathbf{i}$ & 88 \\
\hline 10 & Et $(\mathbf{1 d})$ & $\mathrm{C}_{6} \mathrm{H}_{5}$ & 20 & $3 \mathbf{j}$ & 82 \\
\hline 11 & Et (1d) & $p$-(Me) $\mathrm{C}_{6} \mathrm{H}_{4}$ & 20 & $3 \mathrm{k}$ & 83 \\
\hline 12 & Et (1d) & $p-(\mathrm{Cl}) \mathrm{C}_{6} \mathrm{H}_{4}$ & 20 & 31 & 80 \\
\hline 13 & 2-furyl (1e) & $\mathrm{C}_{6} \mathrm{H}_{5}$ & 20 & $3 m$ & 62 \\
\hline 14 & 2-furyl (1e) & $p$-(Me) $\mathrm{C}_{6} \mathrm{H}_{4}$ & 20 & $3 n$ & 67 \\
\hline
\end{tabular}

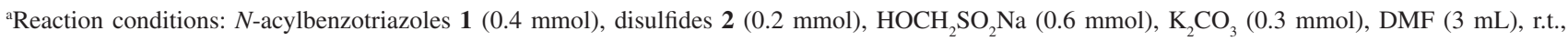
5-30 min. ${ }^{\mathrm{b}}$ Isolated yield.

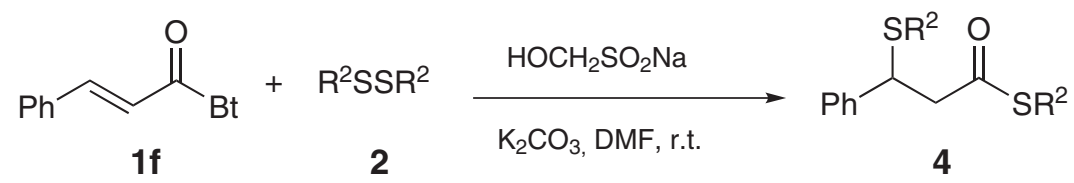<smiles>O=C(CC(Sc1ccccc1)c1ccccc1)Sc1ccccc1</smiles>

$4 a(70 \%)$<smiles>Cc1ccc(SC(=O)CC(Sc2ccc(C)cc2)c2ccccc2)cc1</smiles>

$4 b(68 \%)$<smiles>O=C(CC(Sc1ccc(Cl)cc1)c1ccccc1)Sc1ccc(Cl)cc1</smiles>

$4 c(61 \%)$

Scheme 2. The reaction of cinnamic $N$-acylbenzotriazole with diaryl disulfides. 


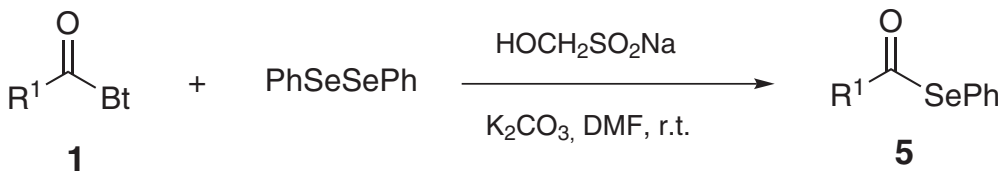<smiles>O=C(c1ccccc1)c1ccccc1</smiles>

$5 a(61 \%)$<smiles>COc1ccc(C(=O)c2ccccc2)cc1</smiles>

$5 b(64 \%)$<smiles>O=C(c1ccccc1)c1ccc(I)cc1</smiles>

$5 c(57 \%)$<smiles>O=C([Se]c1ccccc1)c1ccco1</smiles>

$5 d(51 \%)$

Scheme 3. Synthesis of selenoesters from the reaction of $N$-acylbenzotriazoles with diphenyldiselenide.

transformations. Selenoesters have been used as precursors of acyl radicals ${ }^{19}$ and anions, ${ }^{20}$ mild acyl transfer reagents, ${ }^{21}$ intermediates in the synthesis of ketones, ${ }^{22}$ and for asymmetric aldol reactions. ${ }^{23}$ Under the same conditions, we used diphenyldiselenide as source of selenolate anion, the reaction with $\mathrm{N}$-acylbenzotriazoles afforded the corresponding selenoesters $5 \mathbf{a}-\mathbf{5 d}$ in the presence of Rongalite ${ }^{\circledR}$ and $\mathrm{K}_{2} \mathrm{CO}_{3}$ (Scheme 3). However, acylation of aliphatic diselenide, such as dibenzyl diselenide and dimethyl diselenide, was still unsuccessful under the standard conditions.

In summary, $N$-acylbenzotriazoles have been introduced as new efficient $S$-acylating reagents. The reactions have been demonstrated under mild conditions to give diverse thioesters and selenoesters with moderate to good yield. Rongalite $^{\circledast}$ as an inexpensive promoting reagent for these transformations can be substantiated by short reaction times, which is an additional advantage of this protocol. Efforts to explore the detailed mechanism and further applications of the present system in other transformations using disulfide and diselenide as a reaction partner are ongoing in our group.

\section{Experimental}

Chemicals and solvents were either purchased or synthesized by standard techniques. The reagents of $\mathrm{N}$-acylbenzotriazoles were synthesized by reaction of the corresponding carboxylic acids with $\mathrm{BtH}$ and $\mathrm{SOCl}_{2}$ following the reported one-step general procedure. ${ }^{24}$ Melting points were recorded on Digital Melting Point Apparatus WRS-1B and are uncorrected. ${ }^{1} \mathrm{H}$ NMR and ${ }^{13} \mathrm{C}$ NMR spectra were taken on a Bruker DPX300 spectrometer using $\mathrm{CDCl}_{3}$ or DMSO- $d_{6}$ as the solvent with tetramethylsilane (TMS) as an internal standard at room temperature. Chemical shifts were given in $\delta$ relative to TMS, the coupling constants $J$ are given in Hz. Mass spectrometric analysis was performed on GC-MS analysis (SHIMADZU GCMS-QP2010). Elemental analysis was determined on a Carlo-Erba 1108 instrument.
General procedure for the preparation of thioesters 3,4 and selenoesters 5

A mixture of $N$-acylbenzotriazoles $1(0.4 \mathrm{mmol})$, diaryl disulfides 2 or diphenyldiselenide $(0.2 \mathrm{mmol})$, Rongalite $^{\circledR}$ (3 equiv.), and $\mathrm{K}_{2} \mathrm{CO}_{3}$ (1.5 equiv.) in DMF $(3 \mathrm{~mL})$ was stirred for the corresponding time at room temperature under air. After the reaction was finished, the reaction mixture was washed with water, extracted with ethyl acetate $(3 \times 10 \mathrm{~mL})$, the organic phase was separated and dried over anhydrous sodium sulfate, filtered and the solvent was evaporated under vacuum. The residue was purified by flash column chromatography (ethyl acetate or hexane/ethyl acetate) to afford the desired product thioesters 3 or selenoesters $\mathbf{5}$. If cinnamic $\mathrm{N}$-acylbenzotriazole $\mathbf{1 f}$ was used the reaction substrate, the amount of diaryl disulfides $\mathbf{2}$ is $0.4 \mathrm{mmol}$.

\section{Supplementary Information}

Supplementary data are available free of charge at http://jbcs.sbq.org.br, as PDF file.

\section{Acknowledgments}

We are grateful to the National Key Technology R\&D Program (No. 2007BAI34B00) and the Natural Science Foundation of Zhejiang Province (No. Y4080107) for financial support.

\section{References}

1. Chen, J.; Forsyth, C. J.; Org. Lett. 2003, 5, 1281; Brule, C.; Bouillon, J. P.; Nicolaï, E.; Portella, C.; Synthesis 2003, 436.

2. McGarvey, G. J.; Williams, M.; Hiner, R. N.; Matsubara, Y.; Oh, T.; J. Am. Chem. Soc. 1986, 108, 4943; Conrow, R.; Portoghese, P. S.; J. Org. Chem. 1986, 51, 938; Shimizu, T.; Seki, M.; Tetrahedron Lett. 2002, 43, 1039; Dieter, R. K.; Tetrahedron 1999, 55, 4177; Ikeda, Z.; Hirayama, T.; Matsubara, S.; Angew. 
Chem., Int. Ed. 2006, 45, 8200; Villalobos, J. M.; Srogl, J.; Liebeskind, L. S.; J. Am. Chem. Soc. 2007, 129, 15734.

3. Ozaki, S.; Adachi, M.; Sekiya, S.; Kamikawa, R.; J. Org. Chem. 2003, 68, 4586.

4. Turpin, J. M.; Song, Y.; Inman, J. K.; Huang, M.; Wallqvist, A.; Maynard, A.; Covell, D. G.; Rice, W. G.; Appella, E.; J. Med. Chem. 1999, 42, 67.

5. Detty, M. R.; Wood, G. P.; J. Org. Chem. 1980, 45, 80; Harpp, D. N.; Aida, T.; Chan, T. H.; Tetrahedron Lett. 1979, 31, 2853 Meshram, H. M.; Reddy, G. S.; Bindu, K. H.; Yadav, J. S.; Synthesis 1998, 877; Shah, S. T. A.; Khan, K. M.; Hussain, H.; Hayat, S.; Voelter, W.; Monatsh. Chem. 2005, 136, 1583; Chen, R. E.; Zhang Y. M.; Synth. Commun. 1999, 29, 3699; Polshettiwar, V.; Kaushik, M. P.; Catal. Commun. 2005, 6, 191.

6. Tajima, Y.; Yoshida, A.; Takeda, N.; Oida, S.; Tetrahedron Lett. 1985, 26, 673; Haynes, R. K.; Vonwiller, S. C.; Stokes, J. P.; Merlino, L. M.; Aust. J. Chem. 1988, 41, 881; Choi, J.; Imai, E.; Mihara, M.; Oderaotoshi, Y.; Minakata, S.; Komatsu, M.; J. Org. Chem. 2003, 68, 6164.

7. Brindaban, C. R.; Tanmay, M.; J. Org. Chem. 2004, 69, 5793; Lakouraj, M. M.; Movassagh, B.; Fadaei, Z.; Monatsh. Chem. 2002, 133, 1085.

8. Grieco, P. A.; Yokoyama, Y.; Williams, E.; J. Org. Chem. 1978, 43, 1283.

9. Pittelkow, M.; Kamounah, F. S.; Boas, U.; Pedersen, B.; Christensen, J. B.; Synthesis 2004, 2485.

10. Ravi, D.; Rama Rao, N.; Reddy, G. S. R.; Sucheta, K.; Jayathirtha Rao, V.; Synlett 1994, 856.

11. Imamoto, T.; Kodera, M.; Yokoyama, M.; Synthesis 1982, 134.

12. Katritzky, A. R.; Shestopalov , A. A.; Suzuki, K.; Synthesis 2004, 1806.

13. Cao, H.; McNamee, L.; Alper, H.; J. Org. Chem. 2008, 73, 3530.

14. Arisawa, M.; Kubota, T.; Yamaguchi, M.; Tetrahedron Lett. 2008, 49, 1975.

15. Movassagha, B.; Zakinezhad, Y.; J. Chem. Res. (S) 2006, 369.
16. Sawada, N.; Itoh, T.; Yasuda, N.; Tetrahedron Lett. 2006, 47, 6595.

17. Yang, X. L.; Xu, C. M.; Lin, S. M.; Chen, J. X.; Ding, J. C.; Wu, H. Y.; Su, W. K.; J. Braz. Chem. Soc. 2010, 21, 37; Xiao, H. L.; Chen, J. X.; Liu, M. C.; Zhu, D. J.; Ding, J. C.; Wu, H. Y.; Chem. Lett. 2009, 38, 170; Yang, X. L.; Xu, Y. L.; Chen, J. X.; Ding, J. C.; Wu, H. Y.; Su, W. K.; J. Chem. Res. 2009, 682; Zhu, D. J.; Chen, J. X.; Xiao, H. L.; Liu, M. C.; Ding, J. C.; Wu, H. Y.; Synth. Commun. 2009, 39, 2895; Chen, J. X.; Wu, H. Y.; Jin, C.; Zhang, X. X.; Xie, Y. Y.; Su, W. K.; Green Chem. 2006, 8, 330; Chen, J. X.; Su, W. K.; Wu, H. Y.; Liu, M. C.; Jin, C.; Green Chem. 2007, 9, 972; Su, W. K.; Chen, J. X.; Wu, H. Y.; Jin, C.; J. Org. Chem. 2007, 72, 4524.

18. Guo, W. X.; Chen, J. X.; Wu, D. Z.; Ding, J. C.; Chen, F.; Wu, H. Y.; Tetrahedron 2009, 65, 5240.

19. Keck, G.; Grier, M. C.; Synlett 1999, 1657; Boger, D. L.; Mathvink, R. J.; J. Org. Chem. 1992, 57, 1429; Chen, C.; Crich, D.; Papadatos, A.; J. Am. Chem. Soc. 1992, 114, 8313.

20. Hiiro, T.; Morita, Y.; Inoue, T.; Kambe, N.; Ogawa, A.; Ryu, I.; Sonoda, N.; J. Am. Chem. Soc. 1990, 112, 455.

21. Mukaiyama, T.; Araki, M.; Takei, H.; J. Am. Chem. Soc. 1973, 95, 4763; Anderson, R. J.; Henrick, C. A.; Rosenblum, L. D.; J. Am. Chem. Soc. 1974, 96, 3654; Sviridov, A. F.; Ermolenko, M. S.; Yashunsky, D. V.; Kochetkov, N. K.; Tetrahedron Lett. 1983, 24, 4355.

22. Back, T. G.; Kerr, R. G.; Tetrahedron 1982, 38, 3241; Back, T. G.; Kerr, R. G.; Tetrahedron 1985, 41, 4759.

23. Mukaiyama, T.; Uchiro, H.; Shiina, I.; Kobayashi, S.; Chem. Lett. 1990, 1019; Kobayashi, S.; Uchiro, H.; Fujishita, Y.; Shiina, I.; Mukaiyama, T.; J. Am. Chem. Soc. 1991, 113, 4247; Suh, K. H.; Choo, D. J.; Tetrahedron Lett. 1995, 36, 6109.

24. Katritzky, A. R.; Zhang, Y.; Singh, S. K.; Synthesis 2003, 2795.

Submitted: January 12, 2010

Published online: May 7, 2010 


\title{
An Approach to Synthesis of Thioesters and Selenoesters Promoted by Rongalite ${ }^{\circledR}$
}

\author{
Shao-Miao Lin, ${ }^{a}$ Ji-Lei Zhang, ${ }^{a}$ Jiu-Xi Chen, ${ }^{*, a}$ Wen-Xia Gao, ${ }^{a}$ Jin-Chang Ding, ${ }^{a, b}$ \\ Wei-Ke Su ${ }^{a, c}$ and Hua-Yue $\mathrm{Wu}^{*, a}$ \\ ${ }^{a}$ College of Chemistry and Materials Engineering, Wenzhou University, 325035 Wenzhou, P. R. China \\ ${ }^{b}$ Wenzhou Vocational and Technical College, 325035 Wenzhou, P. R. China \\ 'Zhejiang Key Laboratory of Pharmaceutical Engineering, College of Pharmaceutical Sciences, \\ Zhejiang University of Technology, 310014 Hangzhou, P. R. China
}

\section{Description of the Products}

Compounds 3a-3h, 3j-3m, 3o-3p, 4a-4d are known, compounds $\mathbf{3 i}, \mathbf{3 n}$ and $\mathbf{3 q}$ are new and described below.

\section{S-Phenyl thiobenzoate $(3 \boldsymbol{a})$}

White solid; mp 51-52 ${ }^{\circ} \mathrm{C}$ (Lit. $\left.{ }^{1} \mathrm{mp} 54-58{ }^{\circ} \mathrm{C}\right) ;{ }^{1} \mathrm{H}$ NMR (300 MHz, $\left.\mathrm{CDCl}_{3}\right): \delta$ 7.45-7.62 (m, 8H), 8.02-8.05 (m, 2H); ${ }^{13} \mathrm{C}$ NMR $\left(125 \mathrm{MHz}, \mathrm{CDCl}_{3}\right): \delta 127.49,127.54,128.81$, $129.30,129.55,133.70,135.14,136.75,190.08$.

\section{S-4-Tolyl thiobenzoate $(\mathbf{3} \boldsymbol{b})$}

White solid; mp 70-71 ${ }^{\circ} \mathrm{C}$ (Lit. $\left.{ }^{2} \mathrm{mp} 65-66{ }^{\circ} \mathrm{C}\right) ;{ }^{1} \mathrm{H}$ NMR $\left(300 \mathrm{MHz}, \mathrm{CDCl}_{3}\right): \delta 2.41(\mathrm{~s}, 3 \mathrm{H}), 7.29-7.61(\mathrm{~m}, 7 \mathrm{H})$, 8.01-8.05 (m, 2H); ${ }^{13} \mathrm{C}$ NMR (125 MHz, $\left.\mathrm{CDCl}_{3}\right): \delta 21.29$, $123.71,127.38,128.63,130.02,133.48,134.94,136.64$, $139.71,190.44$.

\section{S-4-Chlorophenyl thiobenzoate (3c)}

White solid; mp 71-72 ${ }^{\circ} \mathrm{C}$ (Lit. $\left.{ }^{3} \mathrm{mp} 73-74{ }^{\circ} \mathrm{C}\right){ }^{1} \mathrm{H}$ NMR (300 MHz, $\left.\mathrm{CDCl}_{3}\right): \delta$ 7.44-7.63 (m, 7H), 8.00-8.03 (m, 2H); ${ }^{13} \mathrm{C}$ NMR $\left(125 \mathrm{MHz}, \mathrm{CDCl}_{3}\right): \delta 125.81,127.47,128.78$, $129.46,133.82,135.94,136.27,136.32,189.55$.

\section{S-Phenyl, 4-methoxythiobenzoate (3d)}

White solid; mp 93-95 ${ }^{\circ} \mathrm{C}$ (Lit. ${ }^{4} \mathrm{mp} 98-99{ }^{\circ} \mathrm{C}$ ); ${ }^{1} \mathrm{H}$ NMR $\left(300 \mathrm{MHz}, \mathrm{CDCl}_{3}\right): \delta 3.80(\mathrm{~s}, 3 \mathrm{H}), 6.88(\mathrm{~d}, J 9 \mathrm{~Hz}, 2 \mathrm{H})$, 7.35-7.44 (m, 5H), 7.93 (d, $J$ 9Hz, 2H); ${ }^{13} \mathrm{C}$ NMR $(125$ $\left.\mathrm{MHz}, \mathrm{CDCl}_{3}\right): \delta 55.55,113.94,127.71,129.17,129.36$, $129.47,129.72,135.19,164.03,188.57$.

\section{S-4-Tolyl 4-methoxythiobenzoate $(3 \boldsymbol{e})^{5}$}

White solid; mp 59-62 ${ }^{\circ} \mathrm{C} ;{ }^{1} \mathrm{H}$ NMR $\left(300 \mathrm{MHz}, \mathrm{CDCl}_{3}\right)$ : $\delta 2.41(\mathrm{~s}, 3 \mathrm{H}), 3.89(\mathrm{~s}, 3 \mathrm{H}), 6.96(\mathrm{~d}, J 9 \mathrm{~Hz}, 2 \mathrm{H}), 7.25-7.28$ $(\mathrm{m}, 2 \mathrm{H}), 7.40(\mathrm{~d}, J 8 \mathrm{~Hz}, 2 \mathrm{H}), 8.01(\mathrm{~d}, J 9 \mathrm{~Hz}, 2 \mathrm{H}) ;{ }^{13} \mathrm{C} \mathrm{NMR}$ $\left(125 \mathrm{MHz}, \mathrm{CDCl}_{3}\right.$ ): $\delta 20.31,54.50,112.87,123.10,128.52$, $128.65,128.99,134.08,138.57,162.93,187.94$.

\section{S-4-Chlorophenyl 4-methoxythiobenzoate $(\mathbf{3 f})$}

White solid; mp 93-94 ${ }^{\circ} \mathrm{C}$ (Lit. ${ }^{6} \mathrm{mp} 98-101{ }^{\circ} \mathrm{C}$ ); ${ }^{1} \mathrm{H}$ NMR (300 MHz, $\mathrm{CDCl}_{3}$ ): $\delta 3.81$ (s, 3H), 6.88-6.91 (m, 2H), 7.35 (m, 4H), $7.92(\mathrm{~d}, J 6.9 \mathrm{~Hz}, 2 \mathrm{H}) ;{ }^{13} \mathrm{C} \mathrm{NMR}$ $\left(125 \mathrm{MHz}, \mathrm{CDCl}_{3}\right): \delta 55.57,114.01,126.22,129.16$, 129.41, 129.77, 135.81, 136.39, 164.18, 188.00.

\section{S-Phenyl 2-iodothiobenzoate $(3 \mathrm{~g})^{7}$}

White solid; mp 54-56 ${ }^{\circ} \mathrm{C} ;{ }^{1} \mathrm{H}$ NMR $\left(300 \mathrm{MHz}, \mathrm{CDCl}_{3}\right)$ : $\delta$ 7.19 (t, 1H), 7.45-7.60 (m, 6H), 7.70-7.73 (m, 1H), 7.95$7.98(\mathrm{~m}, 1 \mathrm{H}) ;{ }^{13} \mathrm{C}$ NMR $\left(125 \mathrm{MHz}, \mathrm{CDCl}_{3}\right): \delta 127.54$, $127.80,128.04,128.59,129.39,129.76,132.44,134.59$, $140.85,142.47,192.32$.

\section{S-4-Tolyl 2-iodothiobenzoate $(\mathbf{3 h})^{8}$}

White solid; mp 60-63 ${ }^{\circ} \mathrm{C}$; ${ }^{1} \mathrm{H}$ NMR $(300 \mathrm{MHz}$, $\left.\mathrm{CDCl}_{3}\right): \delta 2.41(\mathrm{~s}, 3 \mathrm{H}), 7.18(\mathrm{t}, 1 \mathrm{H}), 7.27-7.47(\mathrm{~m}, 5 \mathrm{H})$, $7.71(\mathrm{~d}, J 8 \mathrm{~Hz}, 1 \mathrm{H}), 7.96(\mathrm{~d}, J 8 \mathrm{~Hz}, 1 \mathrm{H}) ;{ }^{13} \mathrm{C} \mathrm{NMR}$ $\left(125 \mathrm{MHz}, \mathrm{CDCl}_{3}\right): \delta 21.54,123.99,128.00,128.57$, $129.98,130.23,132.36,134.55,140.08,140.82,142.58$, 192.80 .

\section{S-4-Chlorophenyl 2-iodothiobenzoate (3i)}

White solid; mp 82-85 ${ }^{\circ} \mathrm{C} ;{ }^{1} \mathrm{H} \mathrm{NMR}\left(300 \mathrm{MHz}, \mathrm{CDCl}_{3}\right)$ : d 7.20-7.21 (m, 1H), 7.43-7.51 (m, 5H), 7.69-7.72 (m, 1H), 7.96-7.99 (m, $1 \mathrm{H}) ;{ }^{13} \mathrm{C}$ NMR $\left(125 \mathrm{MHz}, \mathrm{CDCl}_{3}\right): \delta 91.56$, 125.98, 128.07, 128.61, 129.64, 132.62, 135.82, 136.22, 140.95, 142.08, 191.71. ESI-MS: $\mathrm{m} / z(\%): 376\left([\mathrm{M}+2]^{+}\right.$, 32), $374\left(\mathrm{M}^{+}, 100\right)$. Anal. calc. for $\mathrm{C}_{13} \mathrm{H}_{8}$ ClIOS: $\mathrm{C}, 41.68$; H, 2.15; Found: C, 41.72; H, 2.21. 
S-Phenyl thiopropionate $(3 \mathrm{j})^{9}$

Oil; ${ }^{1} \mathrm{H}$ NMR (300 MHz, $\left.\mathrm{CDCl}_{3}\right): \delta 1.24(\mathrm{t}, J 7.50 \mathrm{~Hz}$, $3 \mathrm{H}), 2.70$ (q, J 7.49 Hz, 2H), 7.42-7.43 (m, 5H); ${ }^{13} \mathrm{C} \mathrm{NMR}$ $\left(125 \mathrm{MHz}, \mathrm{CDCl}_{3}\right): \delta 8.75,36.09,126.91,128.12,128.26$, 133.50, 197.13 .

\section{S-4-Tolyl thiopropionate $(3 \boldsymbol{k})^{3}$}

Oil; ' ${ }^{1} \mathrm{H}$ NMR (300 MHz, $\left.\mathrm{CDCl}_{3}\right): \delta 1.21(\mathrm{t}, J 7.47 \mathrm{~Hz}$, $3 \mathrm{H}), 2.36$ (s, 3H), 2.67 (q, J 7.50 Hz, 2H), $7.19-7.30(\mathrm{~m}$, $4 \mathrm{H}) ;{ }^{13} \mathrm{C}$ NMR $\left(125 \mathrm{MHz}, \mathrm{CDCl}_{3}\right): \delta 9.53,21.20,36.90$, $124.30,129.89,134.39,139.43,198.57$.

\section{S-4-Chlorophenyl thiopropionate $(3 l)^{3}$}

Oil; ${ }^{1} \mathrm{H}$ NMR (300 MHz, $\left.\mathrm{CDCl}_{3}\right): \delta 1.23(\mathrm{t}, J 7.48 \mathrm{~Hz}$, $3 \mathrm{H}), 2.69$ (q, J $7.48 \mathrm{~Hz}, 2 \mathrm{H}), 7.32-7.41(\mathrm{~m}, 4 \mathrm{H}) ;{ }^{13} \mathrm{C} \mathrm{NMR}$ $\left(125 \mathrm{MHz}, \mathrm{CDCl}_{3}\right): \delta 8.43,36.09,126.87,128.12,128.26$, 133.50, 197.16.

\section{S-Phenyl 2-Furancarbothioate (3m)}

White solid; mp 51-52 ${ }^{\circ} \mathrm{C}\left(\right.$ Lit. $\left.{ }^{10} \mathrm{mp} 51-52{ }^{\circ} \mathrm{C}\right) ;{ }^{1} \mathrm{H}$ NMR $\left(300 \mathrm{MHz}, \mathrm{CDCl}_{3}\right): \delta 6.58-6.60(\mathrm{~m}, 1 \mathrm{H}), 7.27(\mathrm{~d}, J 3.6 \mathrm{~Hz}$, $1 \mathrm{H}), 7.46-7.63(\mathrm{~m}, 5 \mathrm{H}), 7.64(\mathrm{~s}, 1 \mathrm{H}) ;{ }^{13} \mathrm{C}$ NMR $(125 \mathrm{MHz}$, $\left.\mathrm{CDCl}_{3}\right): \delta 111.39,115.21,125.23,128.23,128.55,134.12$, $145.45,149.39,177.59$.

\section{S-4-Tolyl 2-Furancarbothioate (3n)}

White solid; $\mathrm{mp} 63-65^{\circ} \mathrm{C} ;{ }^{1} \mathrm{H} \mathrm{NMR}\left(300 \mathrm{MHz}, \mathrm{CDCl}_{3}\right)$ : $\delta 2.41(\mathrm{~s}, 3 \mathrm{H}), 6.57-6.59(\mathrm{~m}, 1 \mathrm{H}), 7.25-7.41(\mathrm{~m}, 5 \mathrm{H}), 7.63$ $(\mathrm{m}, 1 \mathrm{H}) ;{ }^{13} \mathrm{C}$ NMR $\left(125 \mathrm{MHz}, \mathrm{CDCl}_{3}\right): \delta 21.34,112.35$, 116.09, 122.64, 130.10, 135.06, 139.95, 146.37, 150.49, 179.06. ESI-MS: $m / z(\%): 218\left(\mathrm{M}^{+}, 100\right)$. Anal. calc. for $\mathrm{C}_{12} \mathrm{H}_{10} \mathrm{O}_{2} \mathrm{~S}$ : C, 66.03; H, 4.62; Found: C, 60.10; H, 4.59.

\section{S-Phenyl 3-phenyl-3-(phenylthio)propanethioate $(4 a)^{11}$}

White solid; mp 74-76 ${ }^{\circ} \mathrm{C}$; ${ }^{1} \mathrm{H} \mathrm{NMR}\left(300 \mathrm{MHz}, \mathrm{CDCl}_{3}\right)$ : $\delta 3.27(\mathrm{~d}, J 6 \mathrm{~Hz}, 2 \mathrm{H}), 4.75(\mathrm{t}, J 6 \mathrm{~Hz}, 1 \mathrm{H}), 7.23-7.43(\mathrm{~m}$, $15 \mathrm{H}) ;{ }^{13} \mathrm{C}$ NMR $\left(125 \mathrm{MHz}, \mathrm{CDCl}_{3}\right): \delta 49.28,49.42,127.37$, 127.70, 127.82, 128.54, 128.94, 129.18, 129.48, 133.67, 134.39, 139.97, 194.59.

\section{S-4-Tolyl 3-phenyl-3-(phenylthio)propanethioate $(\mathbf{4 b})^{12}$}

White solid; mp 82-83 ${ }^{\circ} \mathrm{C} ;{ }^{1} \mathrm{H}$ NMR $\left(300 \mathrm{MHz}, \mathrm{CDCl}_{3}\right)$ : $\delta 2.34$ (s, 3H), 2.38 (s, 3H), 3.26 (d, J 7.6 Hz, 2H), 4.70 (t, $J$ 7.6 Hz, 1H), 7.07-7.32 (m, 13H); ${ }^{13} \mathrm{C}$ NMR (125 MHz, $\left.\mathrm{CDCl}_{3}\right): \delta 21.10,21.26,49.08,49.44,127.50,127.74$, $128.38,128.91,129.63,129.68,129.99,133.70,134.26$, 138.23, 139.66, 139.96, 194.59 .

S-4-Chlorophenyl 3-(4-chlorophenylthio)-3phenylpropanethioate $(\mathbf{4 c})$

White solid; mp 63-65 ${ }^{\circ} \mathrm{C} ;{ }^{1} \mathrm{H} \mathrm{NMR}\left(300 \mathrm{MHz}, \mathrm{CDCl}_{3}\right.$ ): $\delta 3.24(\mathrm{~d}, J 7.5 \mathrm{~Hz}, 2 \mathrm{H}), 4.69$ (t, $J 7.6 \mathrm{~Hz}, 1 \mathrm{H}), 7.19-7.43$ $(\mathrm{m}, 13 \mathrm{H}) ;{ }^{13} \mathrm{C} \mathrm{NMR}\left(125 \mathrm{MHz}, \mathrm{CDCl}_{3}\right): \delta 49.22,49.43$, 123.9, 125.6, 1207.7, 128.6, 129.4, 130.9, 131.8, 134.2, 135.5, 135.8, 139.5, 141.9, 193.9. ESI-MS: $\mathrm{m} / z$ (\%): 422 $\left([\mathrm{M}+4]^{+}, 10\right), 420\left([\mathrm{M}+2]^{+}, 36\right), 418\left(\mathrm{M}^{+}, 100\right)$. Anal. calc. for $\mathrm{C}_{21} \mathrm{H}_{16} \mathrm{Cl}_{2} \mathrm{OS}_{2}$ : C, 60.14; H, 3.85; Found: C, 60.08; H, 3.94.

\section{Se-Phenyl selenobenzoate (5a)}

Yellow solid; mp 40-41 ${ }^{\circ} \mathrm{C}$ (Lit. ${ }^{12} \mathrm{mp} \mathrm{37-38}{ }^{\circ} \mathrm{C}$ ); ${ }^{1} \mathrm{H}$ NMR $\left(300 \mathrm{MHz}, \mathrm{CDCl}_{3}\right): \delta 7.43-7.52(\mathrm{~m}, 5 \mathrm{H}), 7.60-$ 7.63 (m, 3H), 7.93-7.96 (m, 2H); ${ }^{13} \mathrm{C}$ NMR (125 MHz, $\left.\mathrm{CDCl}_{3}\right): \delta 125.78,127.30,128.91,129.02,129.33,133.84$, $136.29,138.52,192.30$.

\section{Se-phenyl 4-methoxyselenobenzoate (5b)}

White solid; mp 56-58 ${ }^{\circ} \mathrm{C}\left(\right.$ Lit. $\left.{ }^{13} \mathrm{mp} 61-62{ }^{\circ} \mathrm{C}\right) ;{ }^{1} \mathrm{H}$ NMR $\left(300 \mathrm{MHz}, \mathrm{CDCl}_{3}\right): \delta 6.96(\mathrm{~d}, J 6.9 \mathrm{~Hz}, 2 \mathrm{H}), 7.42-7.44(\mathrm{~m}$, $3 \mathrm{H}), 7.59-7.63(\mathrm{~m}, 2 \mathrm{H}), 7.92(\mathrm{~d}, J 6.9 \mathrm{~Hz}, 2 \mathrm{H}) ;{ }^{13} \mathrm{C} \mathrm{NMR}$ $\left(125 \mathrm{MHz}, \mathrm{CDCl}_{3}\right): \delta 55.52,114.05,125.97,128.84$, 129.22, 129.61, 131.25, 136.35, 164.14, 191.20.

\section{Se-Phenyl 2-iodoselenobenzoate (5c)}

Yellow solid; mp 59-60 ${ }^{\circ} \mathrm{C}$ (Lit. $\left.{ }^{14} \mathrm{mp} 76{ }^{\circ} \mathrm{C}\right) ;{ }^{1} \mathrm{H}$ NMR (300 MHz, $\left.\mathrm{CDCl}_{3}\right): \delta$ 7.18-7.18 (m, 1H), 7.43-7.7.45 (m, 4H), 7.63-7.69 (m, 3H), $7.96(\mathrm{~d}, J 7.9 \mathrm{~Hz}, 1 \mathrm{H}) ;{ }^{13} \mathrm{C} \mathrm{NMR}$ $\left(125 \mathrm{MHz}, \mathrm{CDCl}_{3}\right): \delta 90.20,126.65,128.01,128.39$, 129.17, 129.45, 132.53, 135.75, 140.97, 143.76, 195.61.

\section{Se-Phenyl 2-Furancarboselenoate $(\mathbf{5 d})^{15}$}

Oil; ${ }^{1} \mathrm{H}$ NMR (300 MHz, $\left.\mathrm{CDCl}_{3}\right): \delta 6.59-6.60(\mathrm{~m}, 1 \mathrm{H})$, 7.21-7.23 (m, 1H), 7.41-7.44 (m, 3H), 7.58-7.63 (m, 3H); ${ }^{13} \mathrm{C}$ NMR (125 MHz, $\mathrm{CDCl}_{3}$ ): $\delta 112.77,115.18,124.74$, 129.10, 129.32, 136.30, 146.57, 151.74, 180.72.

\section{References}

1. Katritzky, A. R.; Shestopalov, A. A.; Suzuki, K.; Synthesis 2004, 1806.

2. Movassagh, B.; Zakinezhad, Y.; J. Chem. Res. 2006, 369.

3. Barbero, M.; Degani, I.; Dughera, S.; Fochi, R.; Synthesis 2003, 1225.

4. Os'kina, I. A.; Vlasov, V. M.; Russian J. Org. Chem. 2008, 44, 561.

5. Kingsbury, C. A.; Phosphorus, Sulfur Silicon Relat. Elem.1981, 9, 315 .

6. Brindaban, C. R.; Tanmay, M.; J. Org. Chem. 2004, 69, 5793.

7. Nyquist, R. A.; Potts, W. J.; Spectrochim. Acta 1959, 514.

8. Buchholz, G.; Martens, J.; Praefcke, K.; Synthesis 1974, 9, 666.

9. Danheiser, R. L.; Nowick, J. S.; J. Org. Chem. 1991, 56, 1176.

10. Katritzky, A. R.; Shestopalov , A. A.; Suzuki, K.; Synthesis 2004, 1806. 
11. Marzorati, L.; Carlos de Mattos, M.; Wladislaw, B.; Di Vitta, C.; Synth. Commun. 2002, 32, 1427.

12. Marin, G.; Braga, A. L.; Rosa, A. S.; Tetrahedron 2009, 65, 4614.

13. Kato, S.; Kabuto, H.; Ishihara, H.; Murai, T.; Synthesis 1985, 520.
14. Renson, M.; Bulletin des Societes Chimiques Belges 1962, 71, 260.

15. Munbunjong, W.; Lee, E. H.; Ngernmaneerat, P.; Kim, S. J.; Singh, G.; Chavasiri, W.; Jang, D.O.; Tetrahedron 2009, 65, 2467.
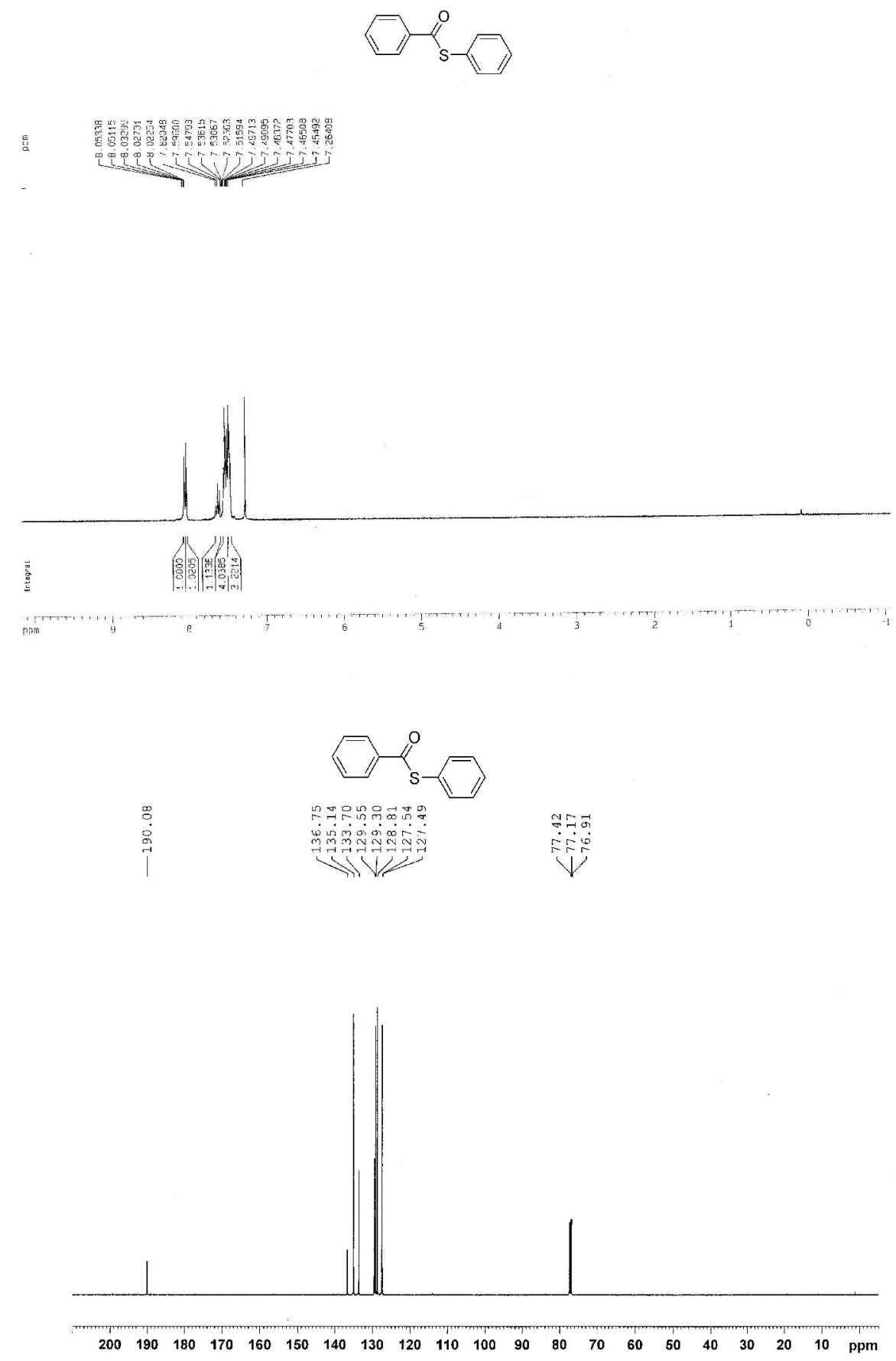

Figure S1. ${ }^{1} \mathrm{H}$ NMR of 3a (300 $\left.\mathrm{MHz}, \mathrm{CDCl}_{3}\right)$ and ${ }^{13} \mathrm{C} \mathrm{NMR} \mathrm{of} \mathrm{3a}\left(125 \mathrm{MHz}, \mathrm{CDCl}_{3}\right)$. 

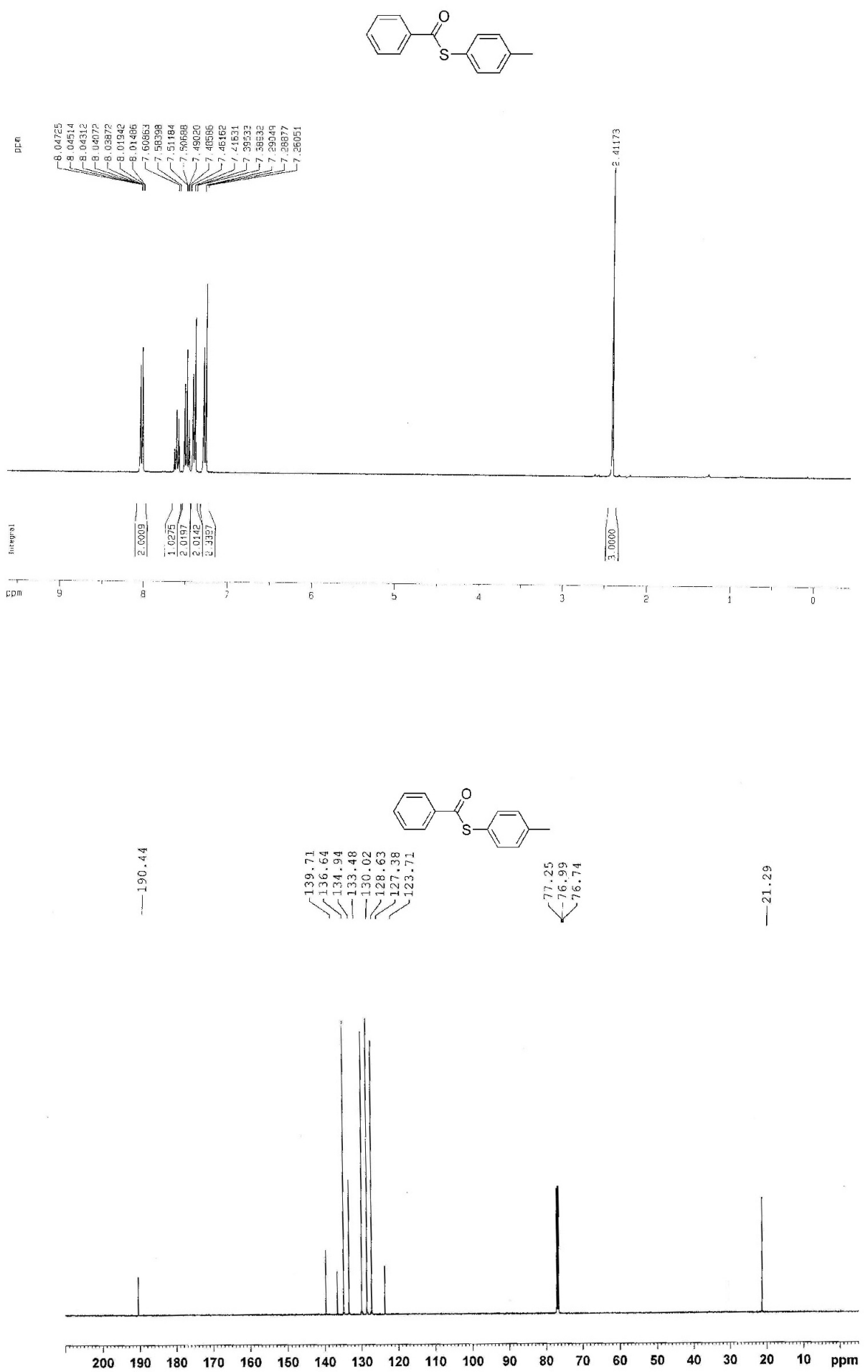

Figure S2. ${ }^{1} \mathrm{H}$ NMR of $\mathbf{3 b}\left(300 \mathrm{MHz}, \mathrm{CDCl}_{3}\right)$ and ${ }^{13} \mathrm{C} \mathrm{NMR}$ of $\mathbf{3 b}\left(125 \mathrm{MHz}, \mathrm{CDCl}_{3}\right)$. 

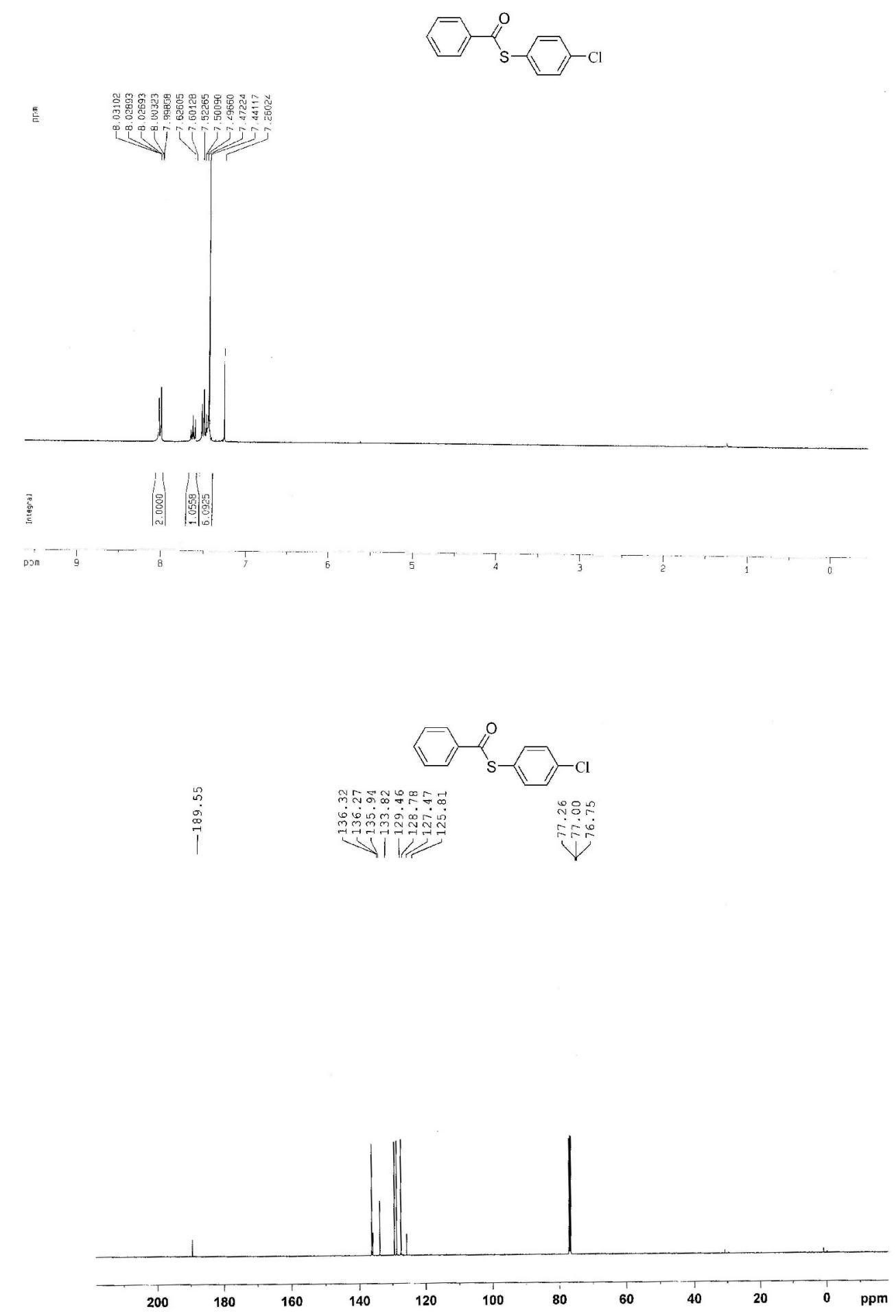

Figure S3. ${ }^{1} \mathrm{H}$ NMR of $\mathbf{3 c}\left(300 \mathrm{MHz}, \mathrm{CDCl}_{3}\right)$ and ${ }^{13} \mathrm{C} \mathrm{NMR} \mathrm{of} \mathbf{3 c}\left(125 \mathrm{MHz}, \mathrm{CDCl}_{3}\right)$. 

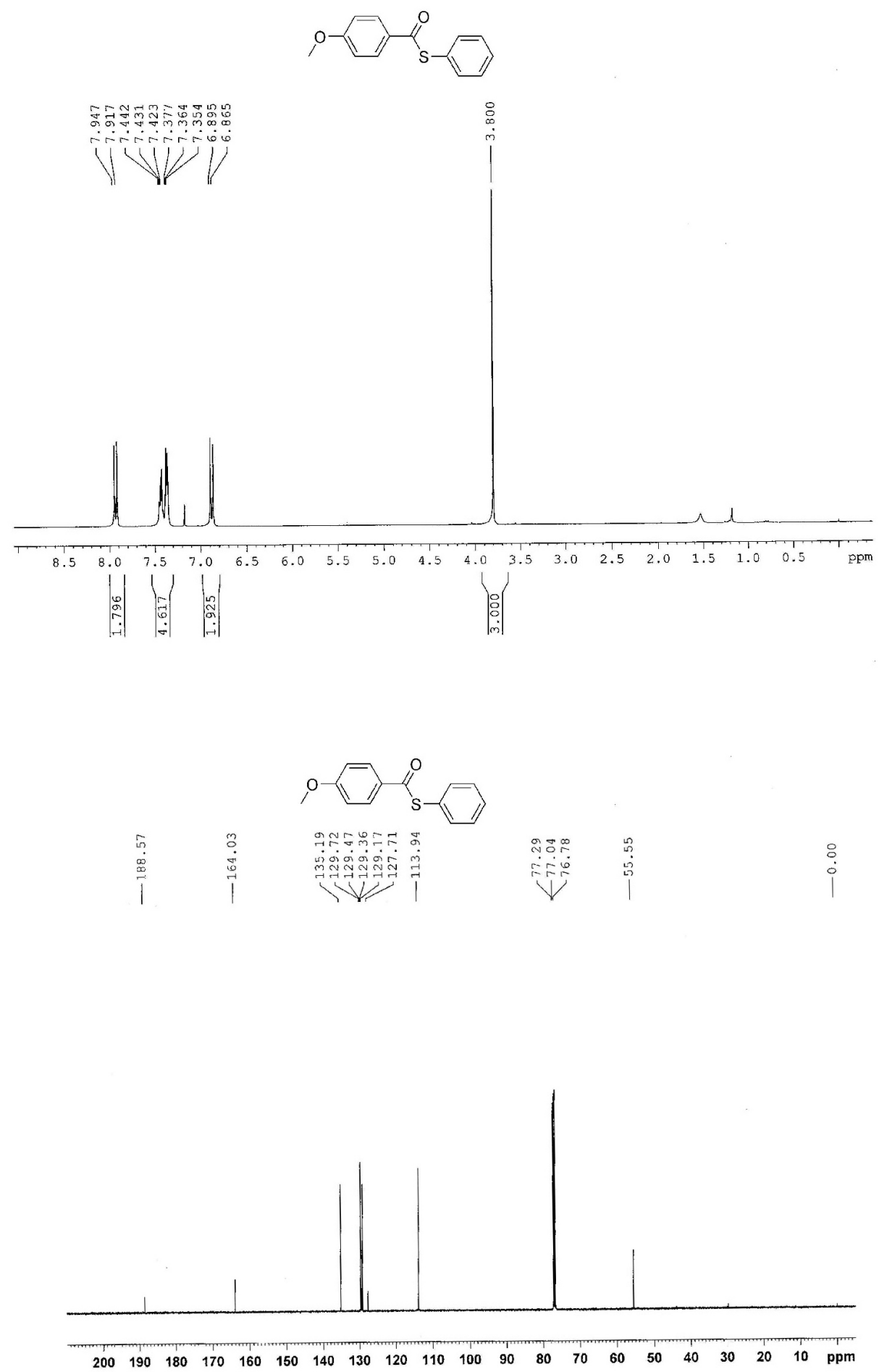

Figure S4. ${ }^{1} \mathrm{H}$ NMR of $\mathbf{3 d}\left(300 \mathrm{MHz}, \mathrm{CDCl}_{3}\right)$ and ${ }^{13} \mathrm{C} \mathrm{NMR}$ of $\mathbf{3 d}\left(125 \mathrm{MHz}, \mathrm{CDCl}_{3}\right)$. 

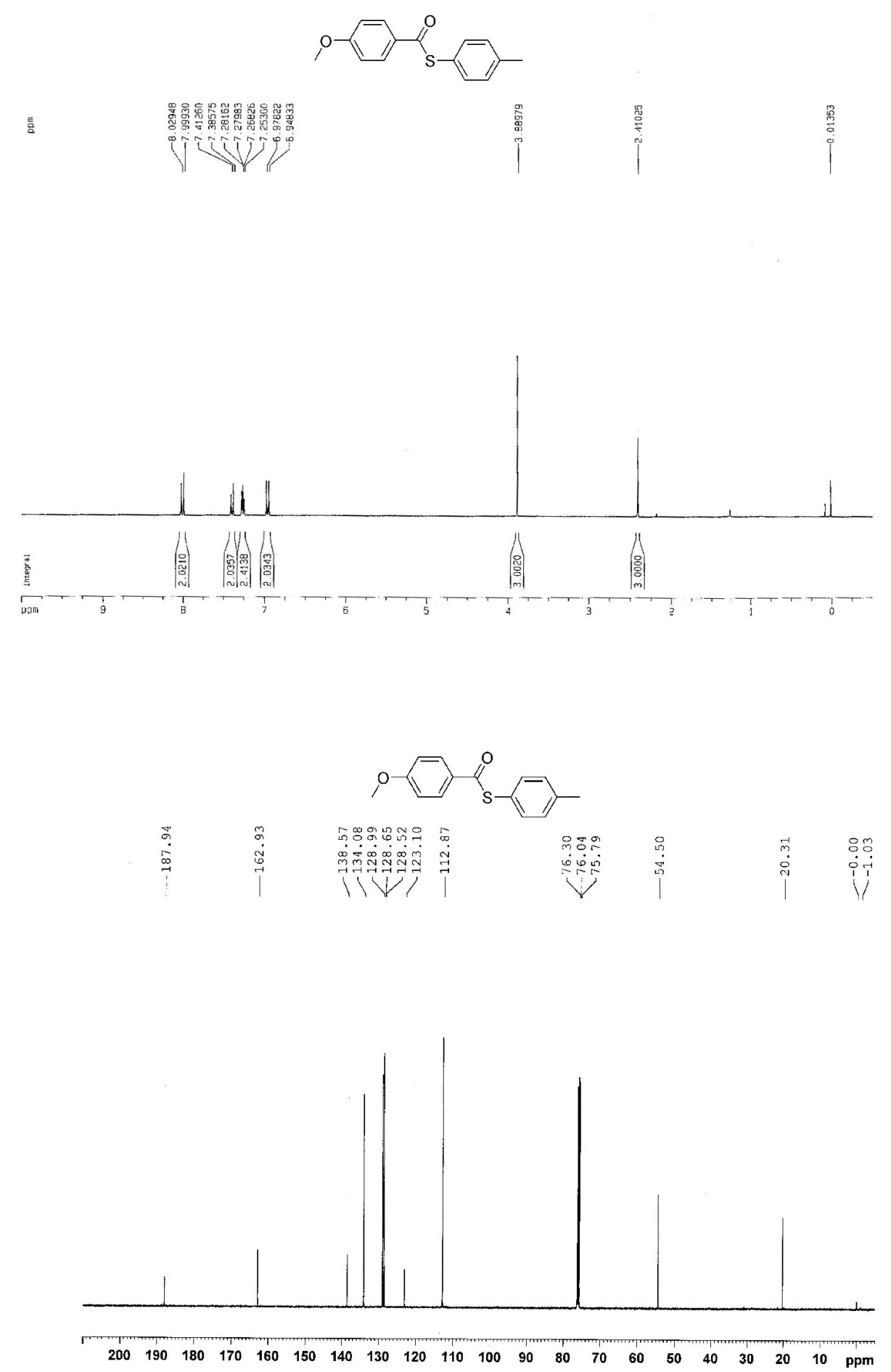

Figure S5. ${ }^{1} \mathrm{H}$ NMR of $\mathbf{3 e}\left(300 \mathrm{MHz}, \mathrm{CDCl}_{3}\right)$ and ${ }^{13} \mathrm{C} \mathrm{NMR}$ of $\mathbf{3 e}\left(125 \mathrm{MHz}, \mathrm{CDCl}_{3}\right)$. 

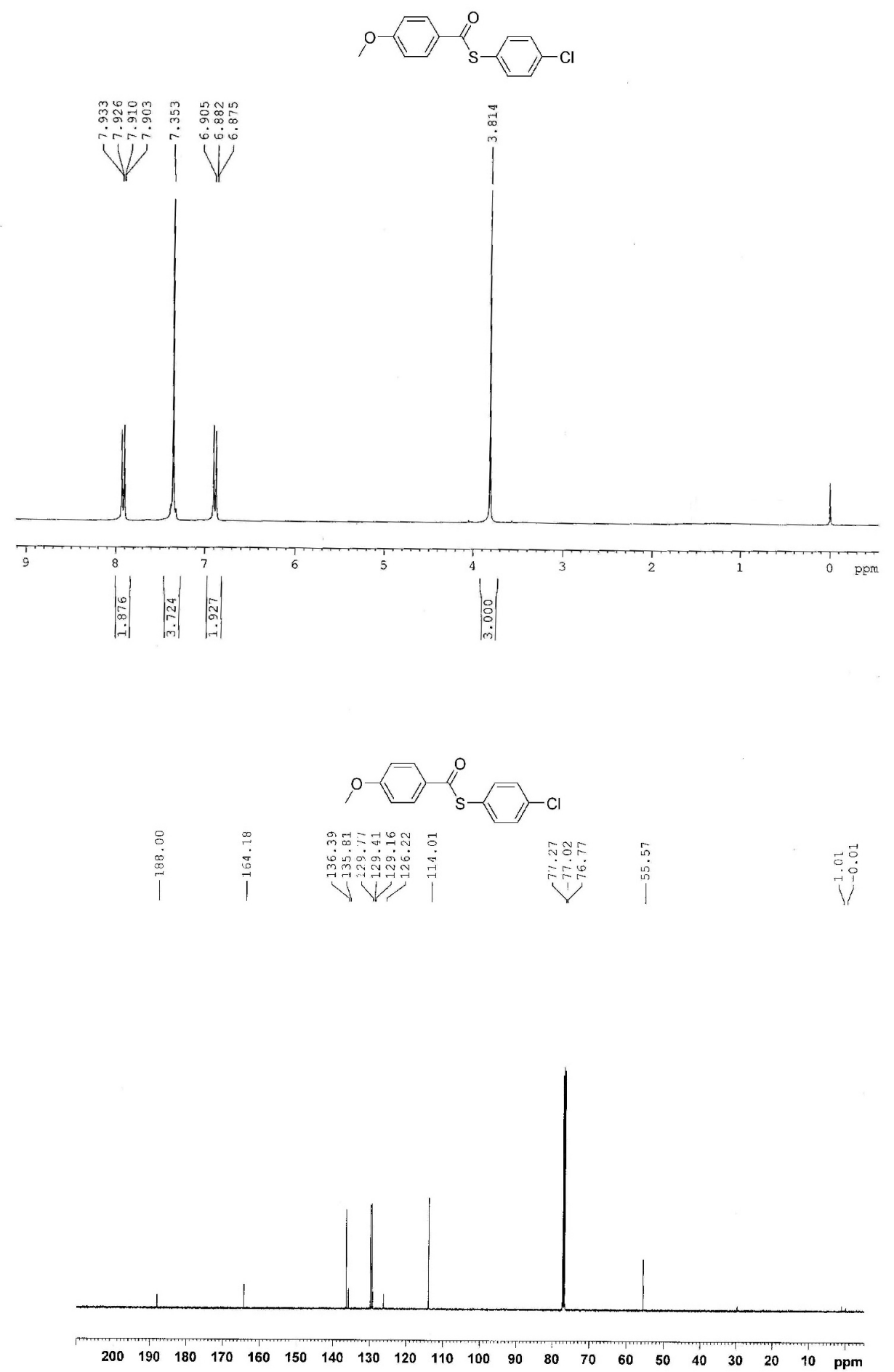

Figure S6. ${ }^{1} \mathrm{H}$ NMR of $\mathbf{3 f}\left(300 \mathrm{MHz}, \mathrm{CDCl}_{3}\right)$ and ${ }^{13} \mathrm{C} \mathrm{NMR}$ of $\mathbf{3 f}\left(125 \mathrm{MHz}, \mathrm{CDCl}_{3}\right)$. 


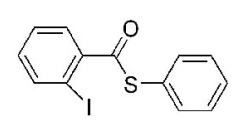

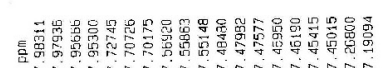

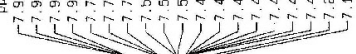
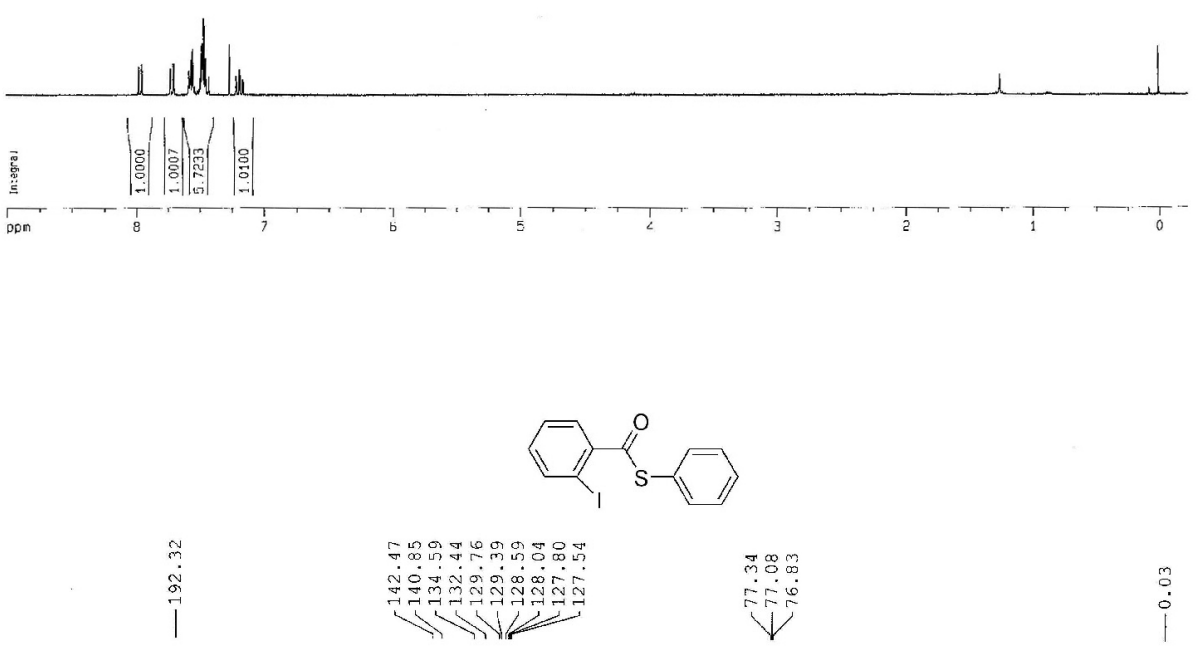

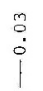

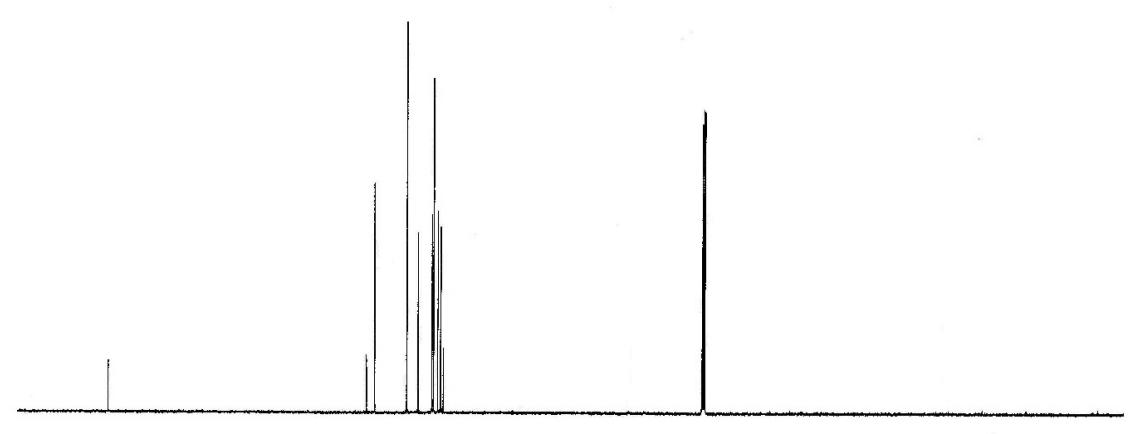

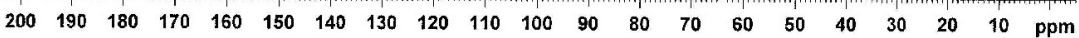

Figure S7. ${ }^{1} \mathrm{H}$ NMR of $\mathbf{3 g}\left(300 \mathrm{MHz}, \mathrm{CDCl}_{3}\right)$ and ${ }^{13} \mathrm{C} \mathrm{NMR} \mathrm{of} \mathbf{3 g}\left(125 \mathrm{MHz}, \mathrm{CDCl}_{3}\right)$. 


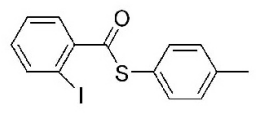

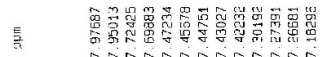

Q i
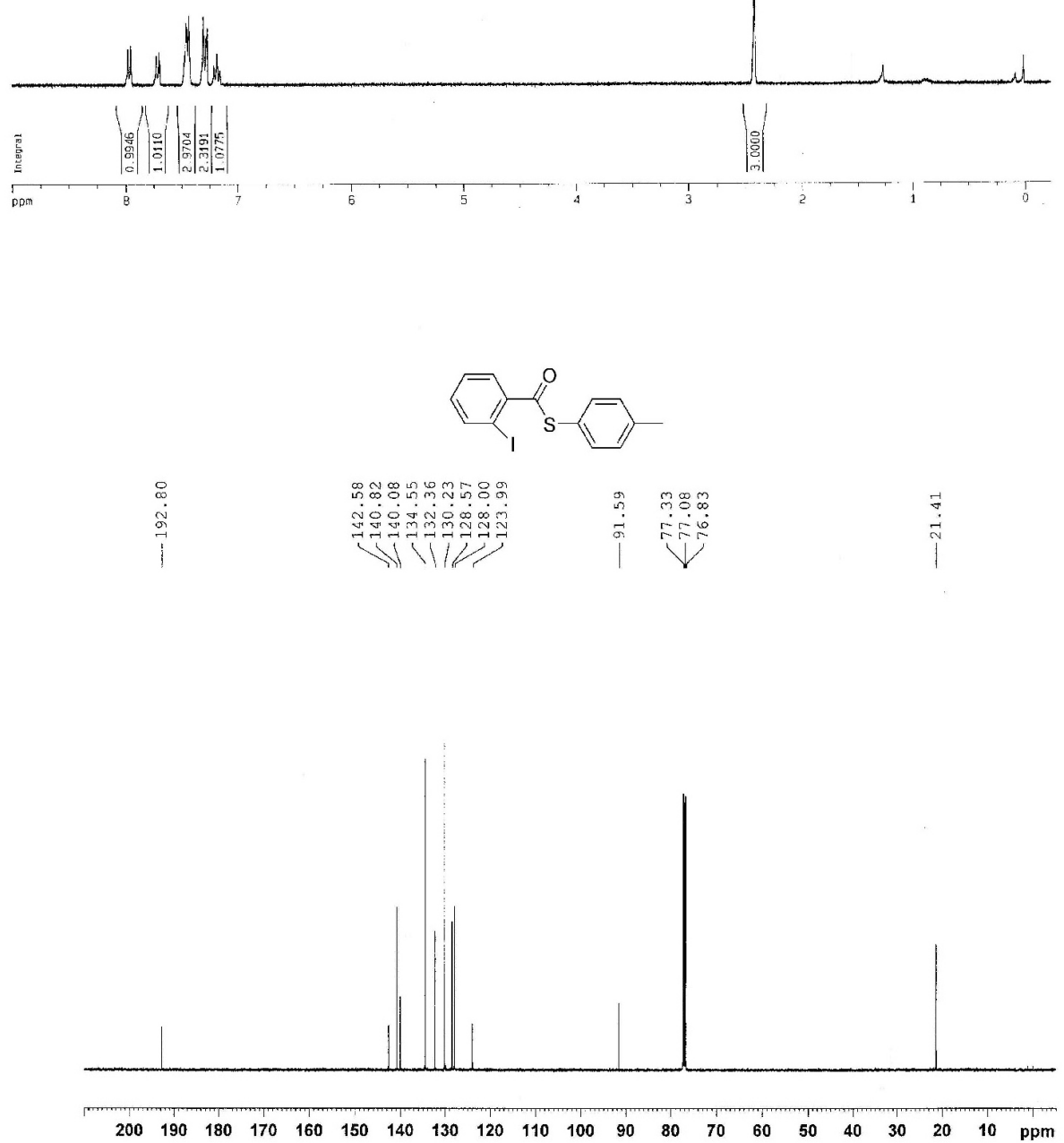

Figure S8. ${ }^{1} \mathrm{H}$ NMR of $\mathbf{3 h}\left(300 \mathrm{MHz}, \mathrm{CDCl}_{3}\right)$ and ${ }^{13} \mathrm{C}$ NMR of $\mathbf{3 h}\left(125 \mathrm{MHz}, \mathrm{CDCl}_{3}\right)$. 

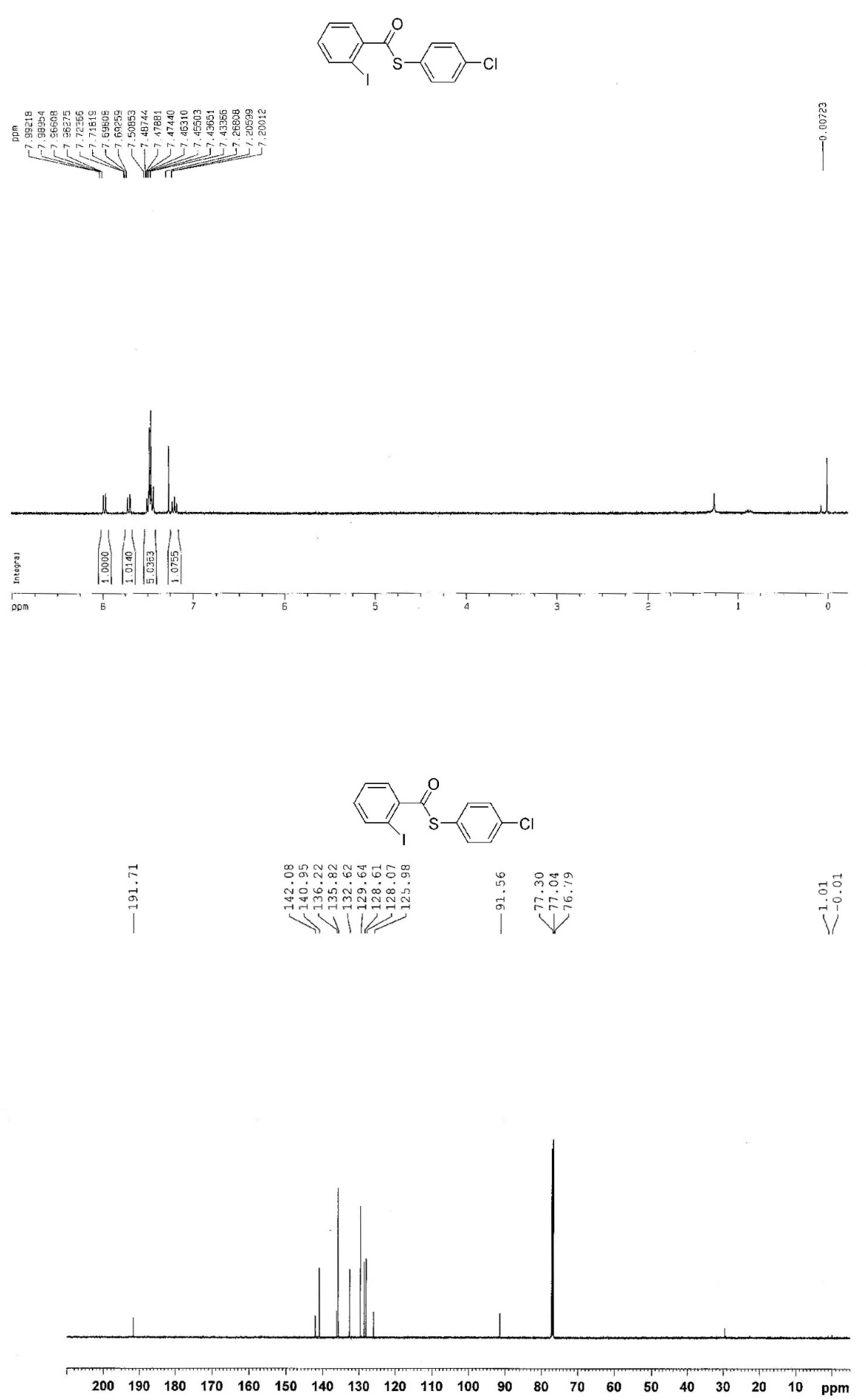

Figure S9. ${ }^{1} \mathrm{H}$ NMR of $\mathbf{3 i}\left(300 \mathrm{MHz}, \mathrm{CDCl}_{3}\right)$ and ${ }^{13} \mathrm{C} \mathrm{NMR}$ of $\mathbf{3 i}\left(125 \mathrm{MHz}, \mathrm{CDCl}_{3}\right)$. 
<smiles>CCC(=O)Sc1ccccc1</smiles>

点

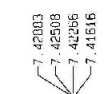

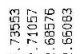

inifi

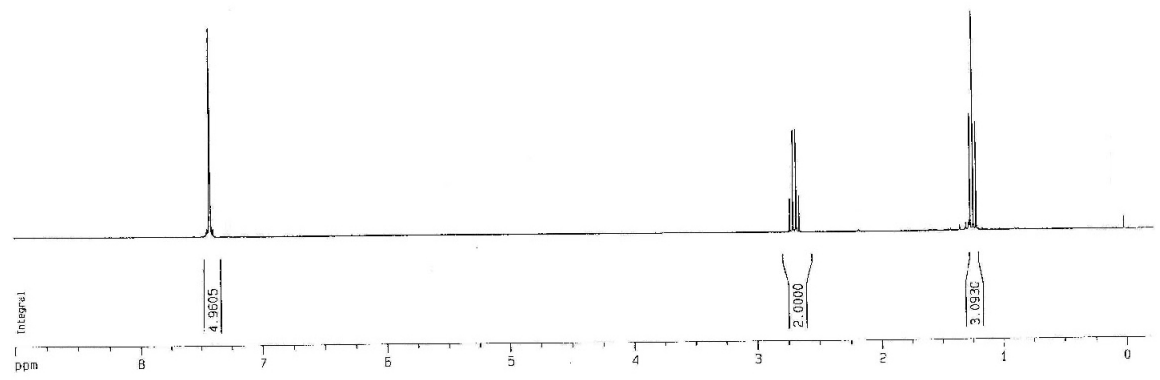

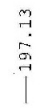

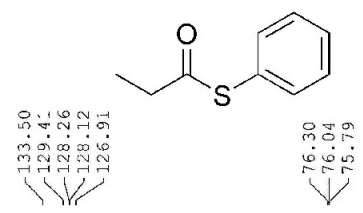

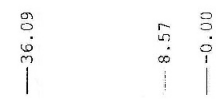

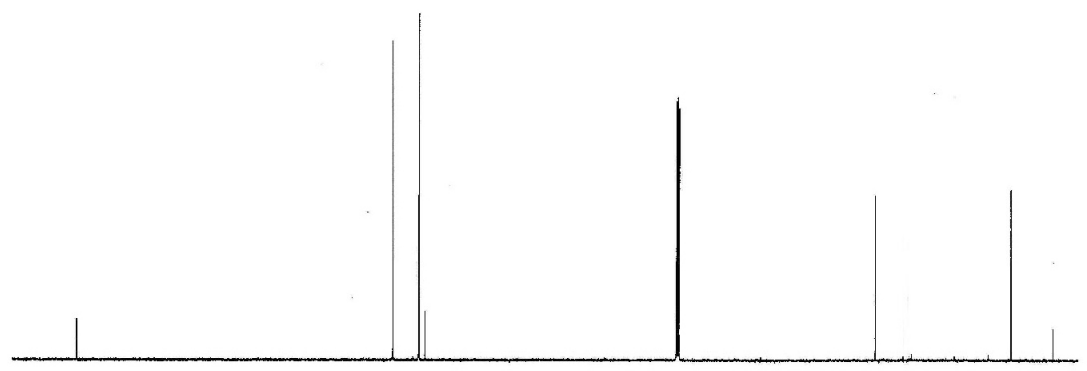

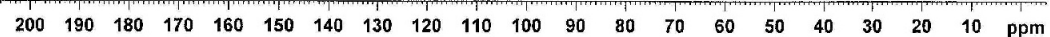

Figure S10. ${ }^{1} \mathrm{H}$ NMR of $\mathbf{3 j}\left(300 \mathrm{MHz}, \mathrm{CDCl}_{3}\right)$ and ${ }^{13} \mathrm{C}$ NMR of $\mathbf{3} \mathbf{j}\left(125 \mathrm{MHz}, \mathrm{CDCl}_{3}\right)$. 
<smiles>CCC(=O)Sc1ccc(C)cc1</smiles>
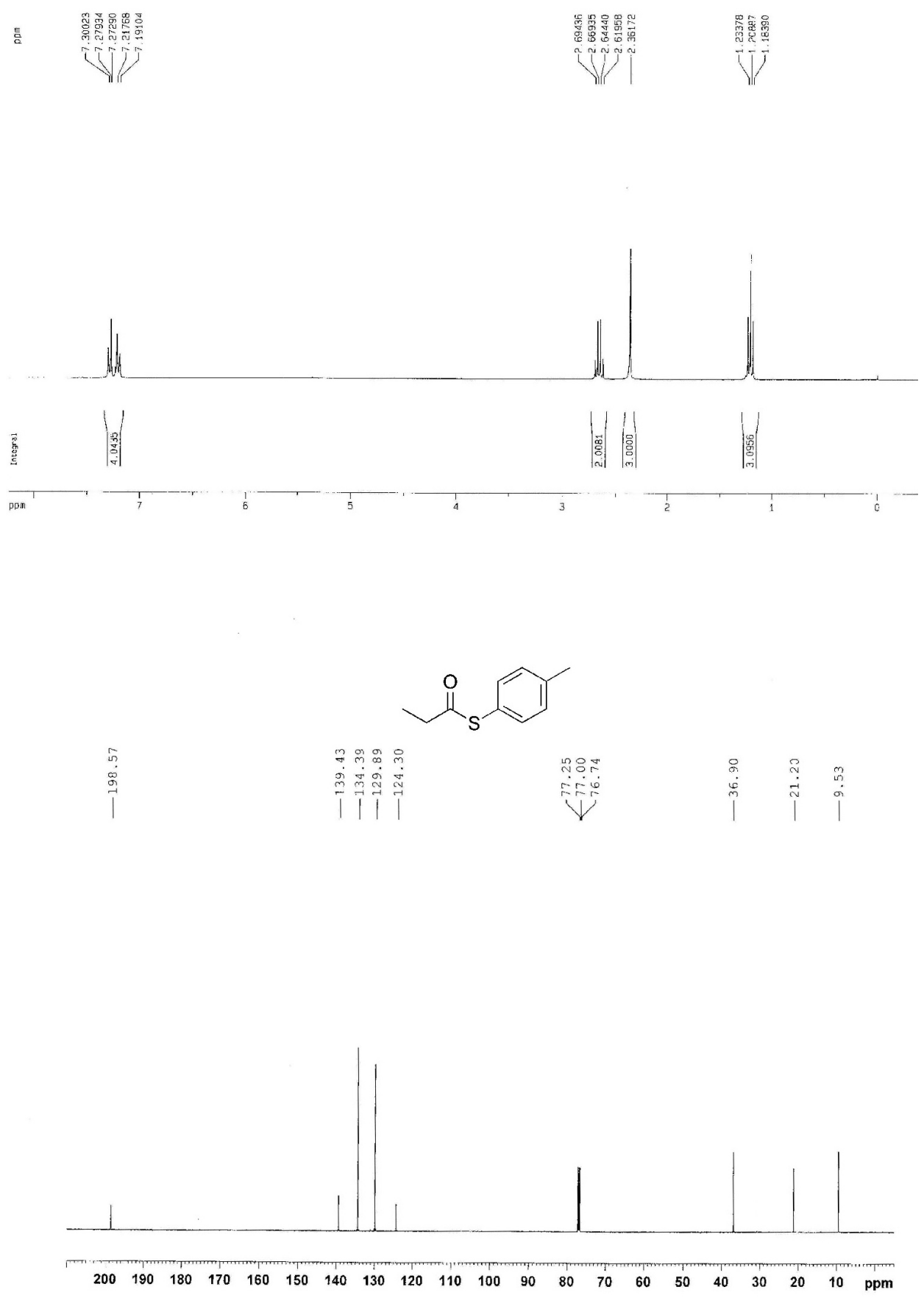

Figure S11. ${ }^{1} \mathrm{H}$ NMR of $\mathbf{3 k}\left(300 \mathrm{MHz}, \mathrm{CDCl}_{3}\right)$ and ${ }^{13} \mathrm{C} \mathrm{NMR}$ of $\mathbf{3 k}\left(125 \mathrm{MHz}, \mathrm{CDCl}_{3}\right)$. 


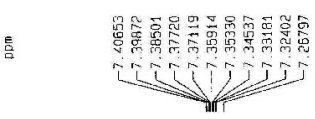<smiles>CCC(=O)Sc1ccc(Cl)cc1</smiles> 

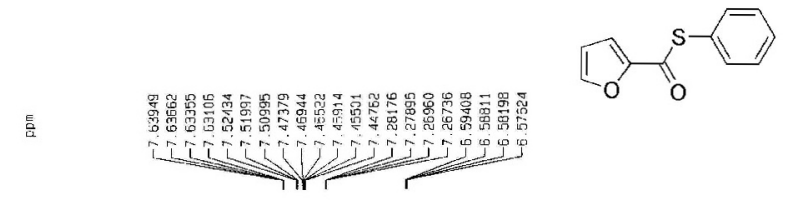

o 0 
言
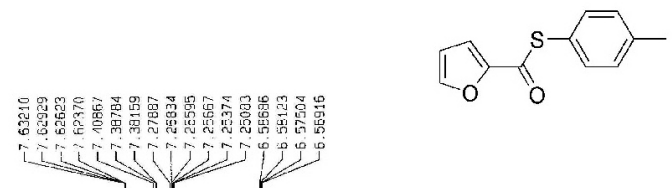
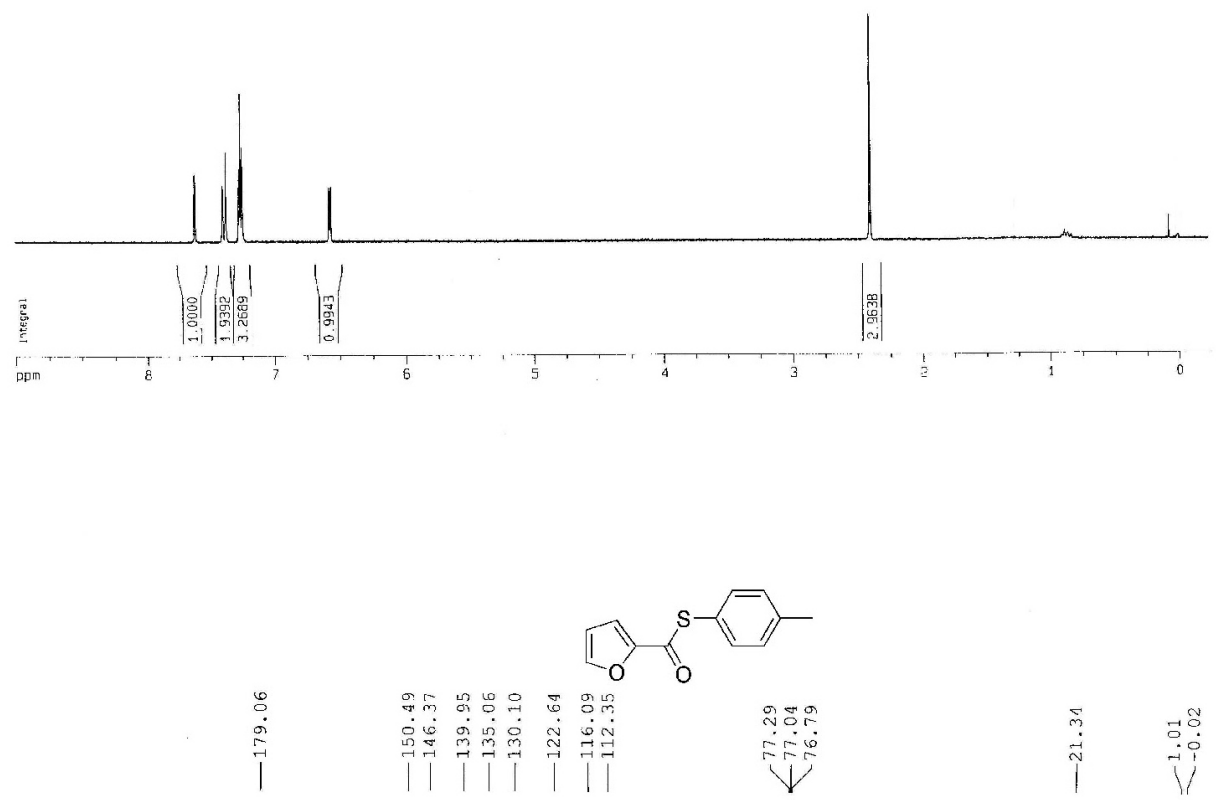

iे

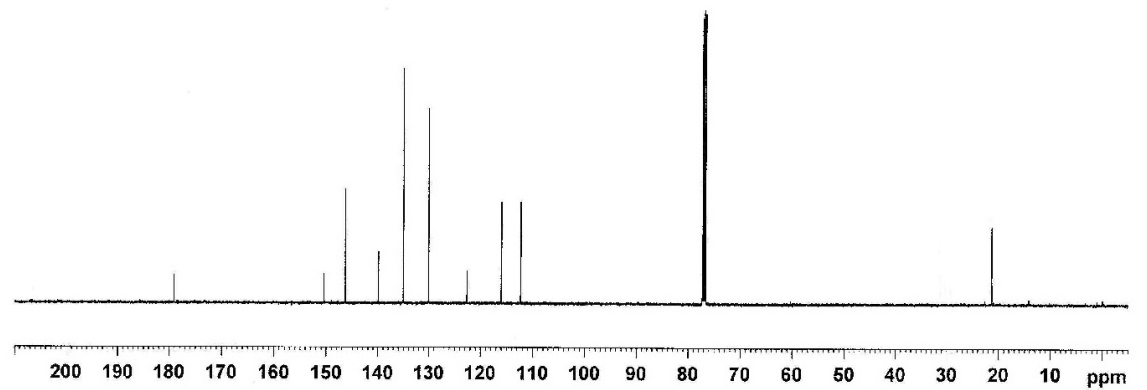

Figure S14. ${ }^{1} \mathrm{H}$ NMR of $\mathbf{3 n}\left(300 \mathrm{MHz}, \mathrm{CDCl}_{3}\right)$ and ${ }^{13} \mathrm{C} \mathrm{NMR}$ of $\mathbf{3 n}\left(125 \mathrm{MHz}, \mathrm{CDCl}_{3}\right)$. 

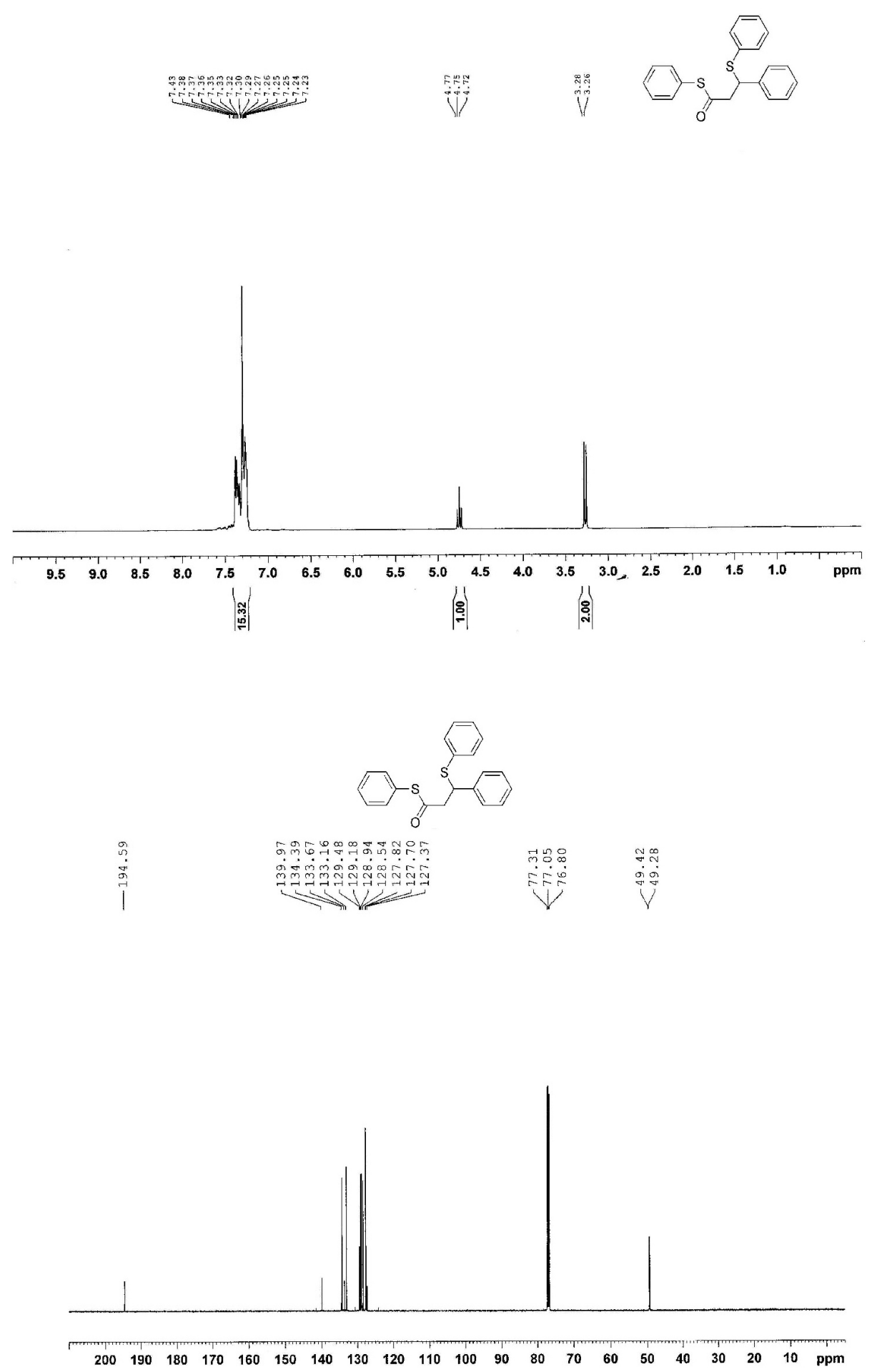

Figure S15. ${ }^{1} \mathrm{H} \mathrm{NMR}$ of $\mathbf{4 a}\left(300 \mathrm{MHz}, \mathrm{CDCl}_{3}\right)$ and ${ }^{13} \mathrm{C} \mathrm{NMR}$ of $\mathbf{4 a}\left(125 \mathrm{MHz}, \mathrm{CDCl}_{3}\right)$. 

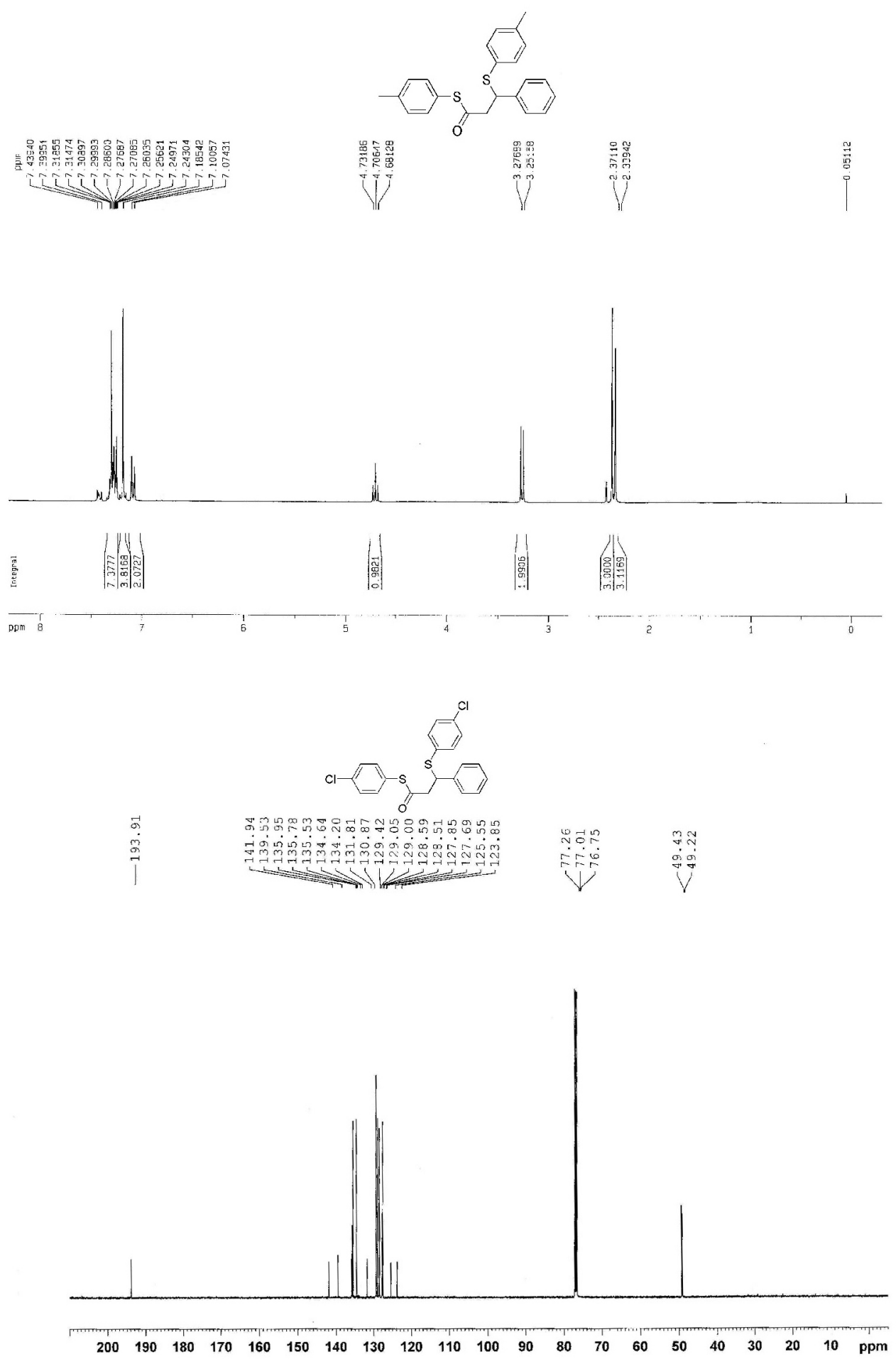

Figure S16. ${ }^{1} \mathrm{H}$ NMR of $\mathbf{4 b}\left(300 \mathrm{MHz}, \mathrm{CDCl}_{3}\right)$ and ${ }^{13} \mathrm{C} \mathrm{NMR} \mathrm{of} \mathbf{4 b}\left(125 \mathrm{MHz}, \mathrm{CDCl}_{3}\right)$. 

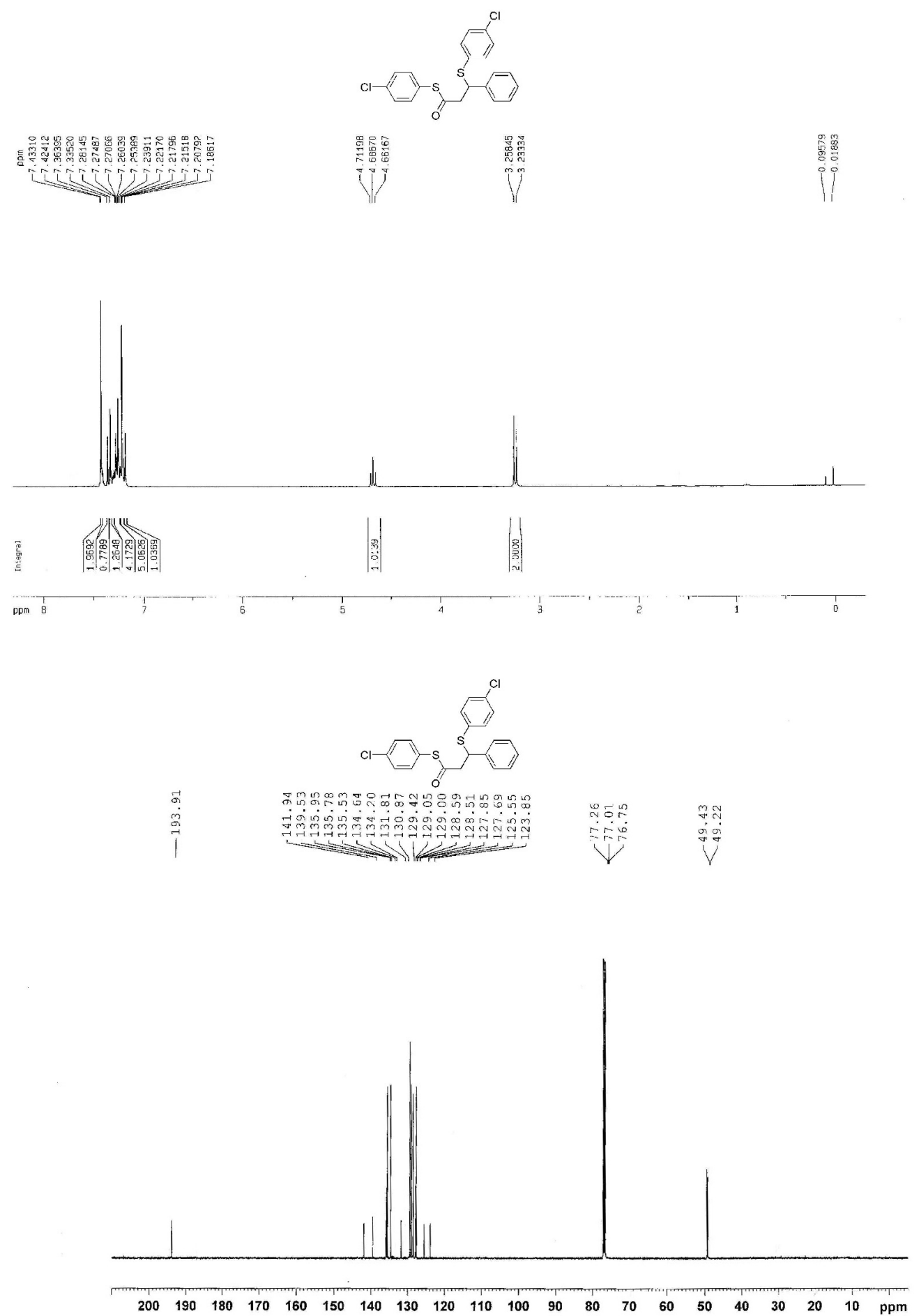

Figure S17. ${ }^{1} \mathrm{H} \mathrm{NMR}$ of $\mathbf{4 c}\left(300 \mathrm{MHz}, \mathrm{CDCl}_{3}\right)$ and ${ }^{13} \mathrm{C} \mathrm{NMR}$ of $\mathbf{4 c}\left(125 \mathrm{MHz}, \mathrm{CDCl}_{3}\right)$. 

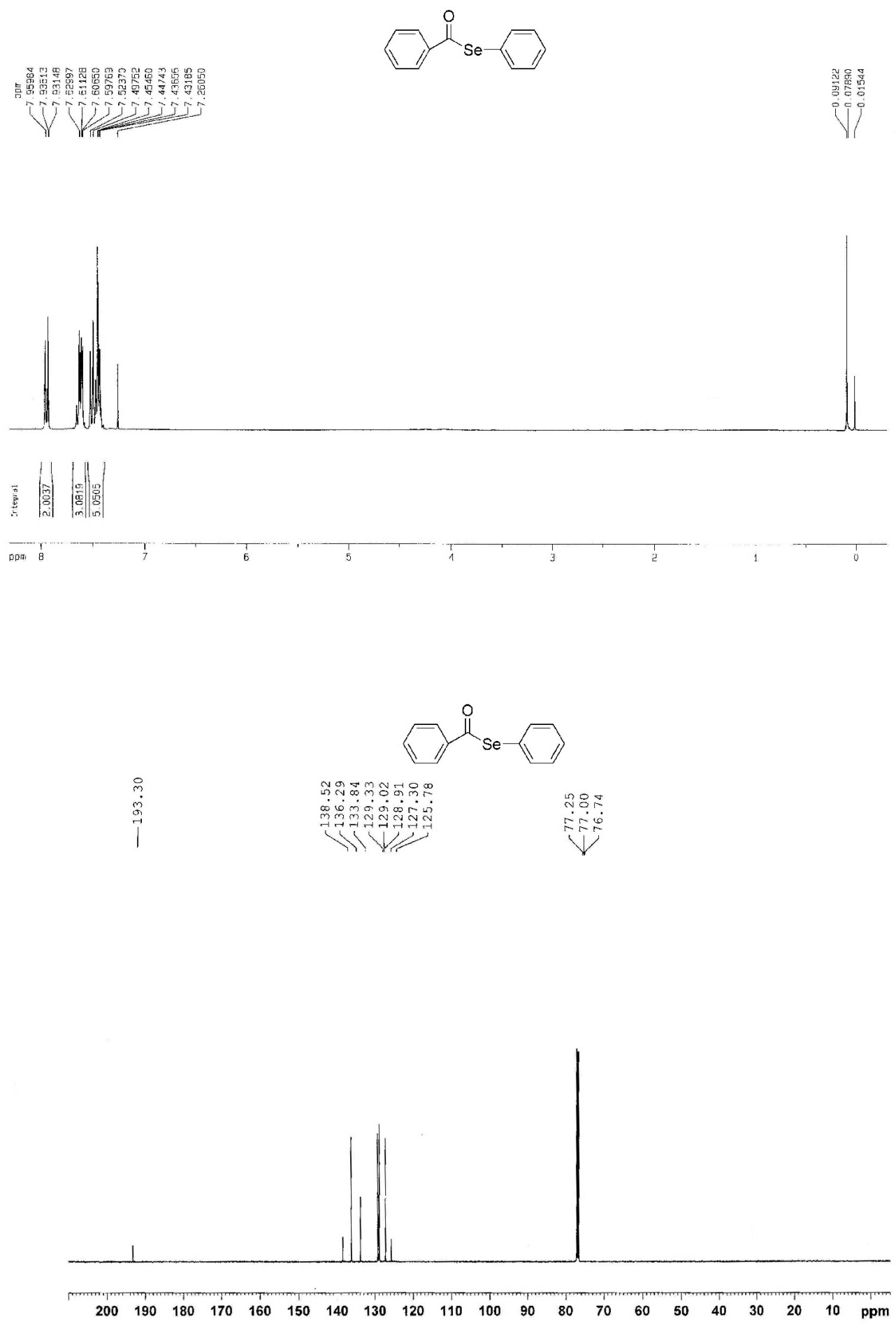

Figure S18. ${ }^{1} \mathrm{H} \mathrm{NMR}$ of $\mathbf{5 a}\left(300 \mathrm{MHz}, \mathrm{CDCl}_{3}\right)$ and ${ }^{13} \mathrm{C} \mathrm{NMR}$ of $\mathbf{5 a}\left(125 \mathrm{MHz}, \mathrm{CDCl}_{3}\right)$. 

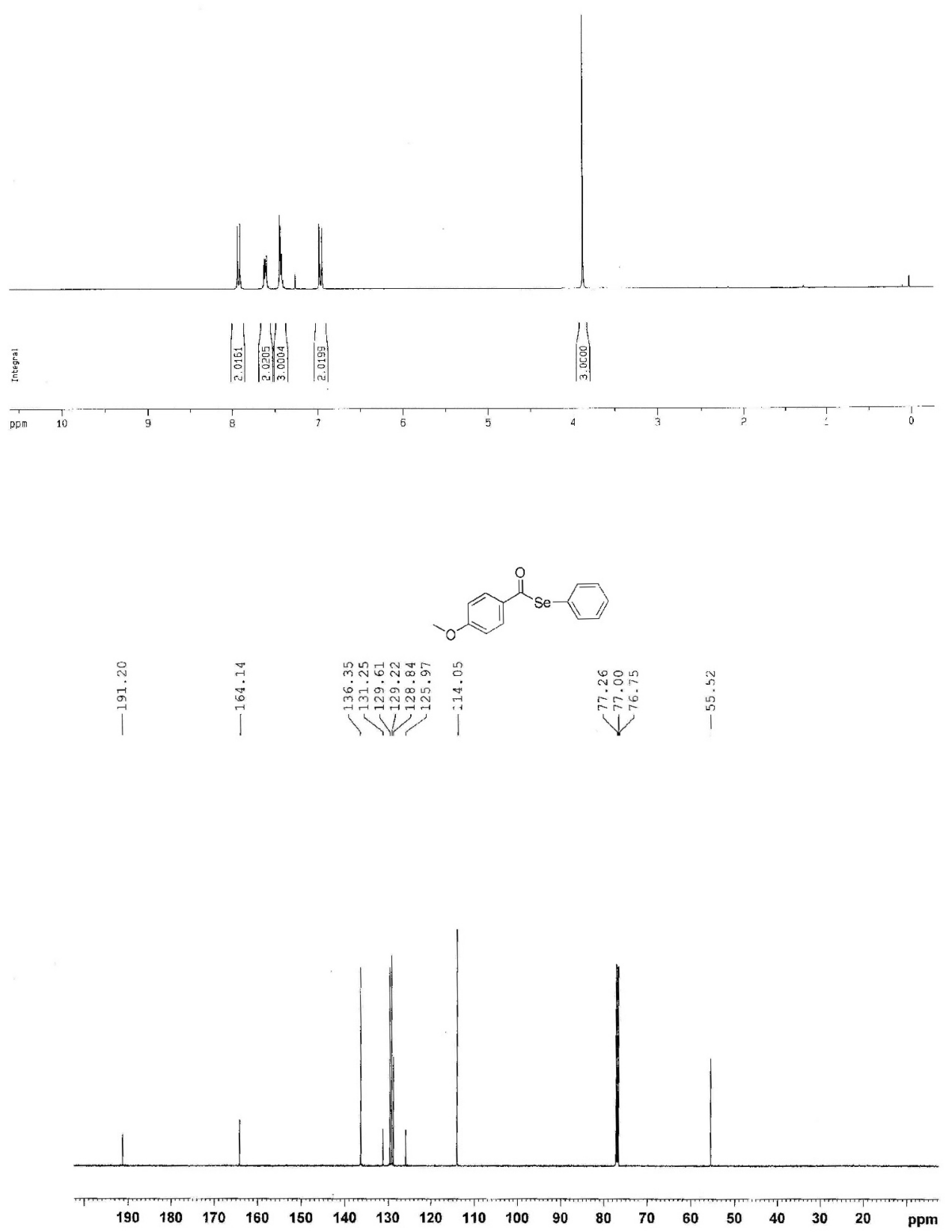

Figure S19. ${ }^{1} \mathrm{H}$ NMR of $\mathbf{5 b}\left(300 \mathrm{MHz}, \mathrm{CDCl}_{3}\right)$ and ${ }^{13} \mathrm{C} \mathrm{NMR}$ of $\mathbf{5 b}\left(125 \mathrm{MHz}, \mathrm{CDCl}_{3}\right)$. 
<smiles>O=C(O)c1ccccc1I</smiles>

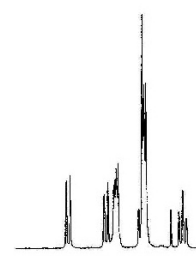

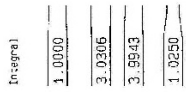

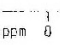
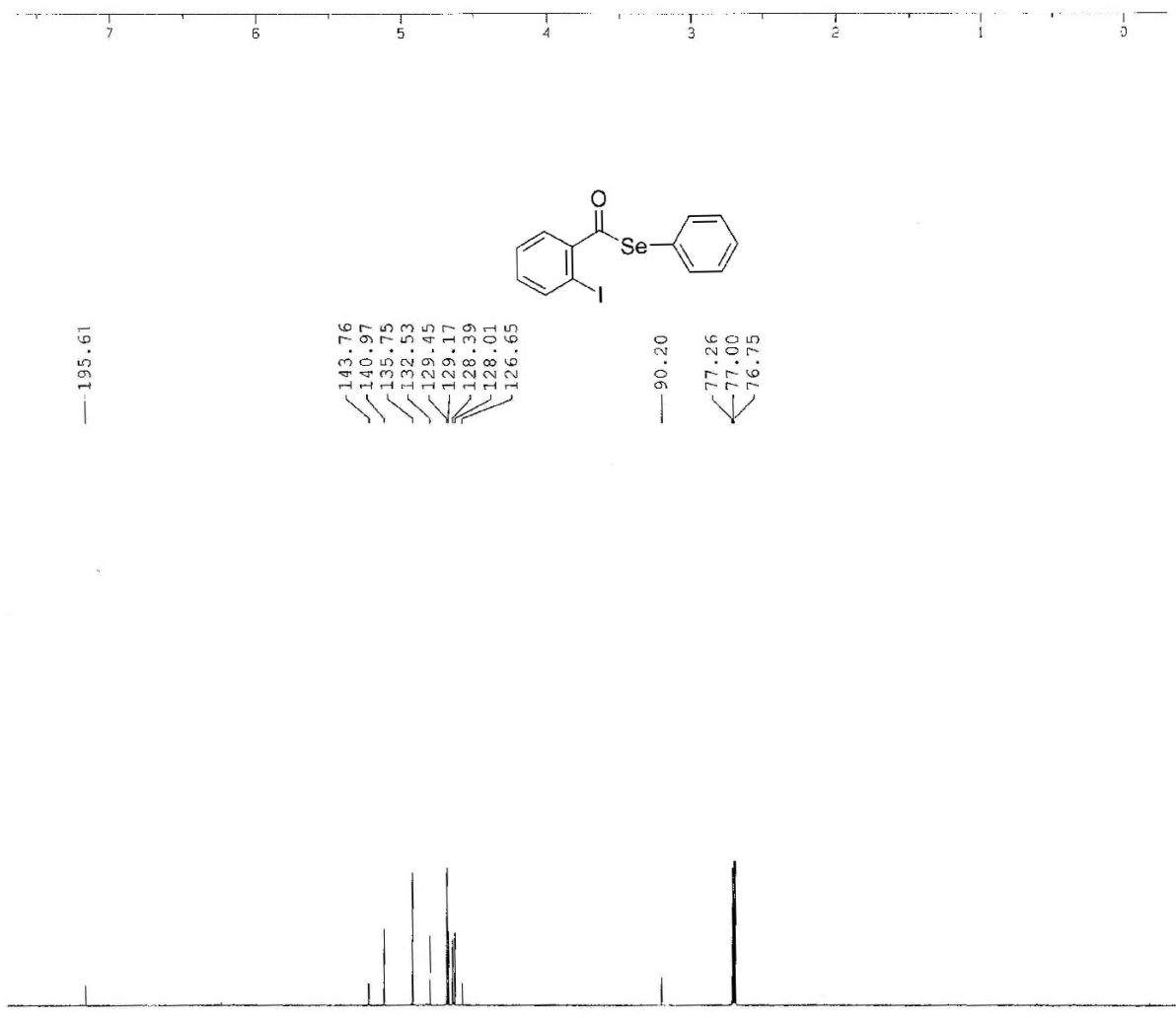

$\begin{array}{lllllllllllllllllllll}200 & 190 & 180 & 170 & 160 & 150 & 140 & 130 & 120 & 110 & 100 & 90 & 80 & 70 & 60 & 50 & 40 & 30 & 20 & 10 & \mathrm{ppm}\end{array}$

Figure S20. ${ }^{1} \mathrm{H}$ NMR of $\mathbf{5 c}\left(300 \mathrm{MHz}, \mathrm{CDCl}_{3}\right)$ and ${ }^{13} \mathrm{C} \mathrm{NMR}$ of $\mathbf{5 c}\left(125 \mathrm{MHz}, \mathrm{CDCl}_{3}\right)$. 

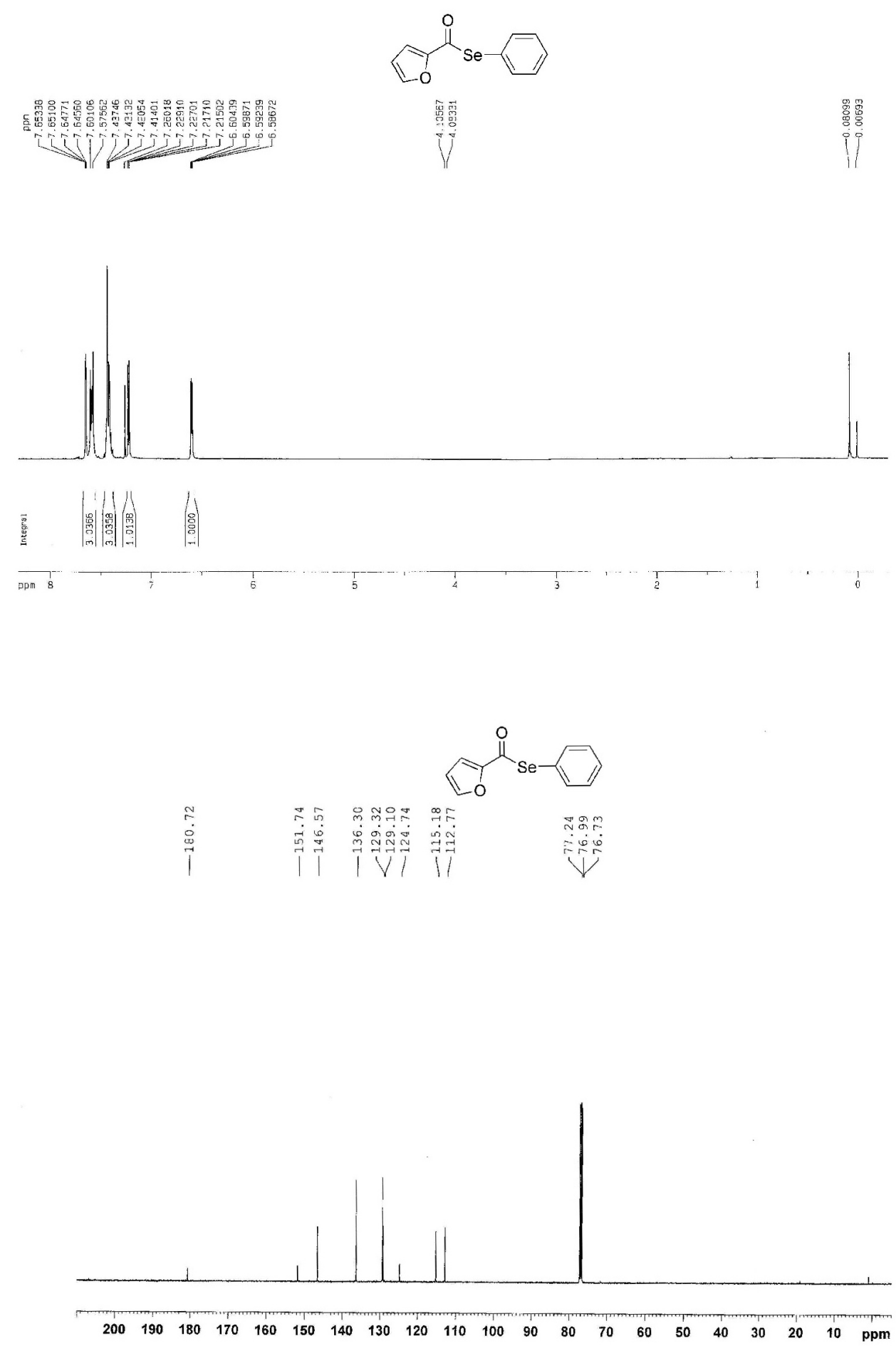

Figure S21. ${ }^{1} \mathrm{H}$ NMR of $\mathbf{5 d}\left(300 \mathrm{MHz}, \mathrm{CDCl}_{3}\right)$ and ${ }^{13} \mathrm{C}$ NMR of $\mathbf{5 d}\left(125 \mathrm{MHz}, \mathrm{CDCl}_{3}\right)$. 